\title{
tRNA-derived small RNAs: A novel class of small RNAs in human hypertrophic scar fibroblasts
}

\author{
YAPING ZHANG ${ }^{1,2}$, QIN DENG ${ }^{1,2}$, LONGXIANG TU $^{1}$, DAN LV $^{1,2}$ and DEWU LIU ${ }^{1}$ \\ ${ }^{1}$ Institute of Burns, The First Affiliated Hospital of Nanchang University; ${ }^{2}$ Department of Medicine, \\ Graduate School of Nanchang University, Nanchang, Jiangxi 330006, P.R. China
}

Received May 7, 2019; Accepted October 14, 2019

DOI: $10.3892 /$ ijmm.2019.4411

\begin{abstract}
RNA-derived small RNAs (tsRNAs) have been shown to play regulatory roles in many physiological and pathological processes. However, their roles in hypertrophic scars remain unclear. The present study investigated differentially expressed tsRNAs in human hypertrophic scar fibroblasts and normal skin fibroblasts via high-throughput sequencing. Several dysregulated tsRNAs were validated by reverse transcription-quantitative polymerase chain reaction (RT-qPCR). Gene Ontology (GO) and Kyoto Encyclopedia of Genes and Genomes (KEGG) pathway enrichment, target prediction, coexpression networks and competing endogenous RNA (ceRNA) networks were evaluated to discover the principal functions of significantly differentially expressed tsRNAs. In total, 67 differentially expressed tsRNAs were detected, of which 27 were upregulated and 40 downregulated in hypertrophic scar fibroblasts. The GO analysis indicated that the dysregulated tsRNAs are associated with numerous biological functions, including 'nervous system development', 'cell adhesion', 'focal adhesion', 'protein binding', 'angiogenesis' and 'actin binding'. KEGG pathway analysis revealed that the most altered pathways include 'Ras signaling pathway', 'Rap1 signaling pathway' and 'cGMP-PKG signaling pathway'. The target genes of the differentially expressed tsRNAs participate in several signaling pathways important for scar formation. The results of RT-qPCR were consistent with those of sequencing. MicroRNA (miR)-29b-1-5p was identified as a target of tsRNA-23678 and was downregulated in hypertrophic scar fibroblasts, constituting a negative regulatory factor for scar formation. Furthermore, tsRNA-23761 acted as a ceRNA and bound to miR-3135b to regulate the expression of miR-3135b targets, including angiotensin-converting enzyme. Collectively, these findings reveal that tsRNAs are differentially expressed
\end{abstract}

Correspondence to: Professor Dewu Liu, Institute of Burns, The First Affiliated Hospital of Nanchang University, 17 Yongwaizheng Street, Nanchang, Jiangxi 330006, P.R. China

E-mail: dewuliu@126.com

Key words: tRNA-derived small RNA, fibroblasts, hypertrophic scar, wound healing, RNA sequencing in human hypertrophic scar fibroblasts, and may contribute to the molecular mechanism and treatment of hypertrophic scars.

\section{Introduction}

Scar is a generic term that refers to the histopathological changes in normal skin tissue caused by various types of trauma, and scars are an inevitable product in the process of human trauma repair (1). After skin damage, normal scars are flat, thin and almost invisible. However, factors such as excessive deposition of extracellular matrix (ECM) and excessive proliferation of fibroblasts can lead to the formation of proliferative scars (2-4). A hypertrophic scar has a protruding surface, and is irregularly shaped with an uneven appearance, red color and solid texture, with burning and itching sensations on the skin surface (5). Hypertrophic scars can damage the function and appearance of the skin and impact the patient's psychology and physiology (6-9). Although proliferative scars can be treated with surgery, radiotherapy, steroid injection and other means, these treatments have not been optimized, and the molecular mechanisms of proliferative scars remain unknown (10).

Recent studies have discovered a new class of small RNAs known as tRNA-derived small RNAs (tsRNAs) that are different from typical noncoding RNA. tsRNAs are fragments derived from transfer RNA (tRNA). tsRNAs can be divided into two main types according to the length of the tRNA and the cleavage site: The first is the stress-induced tRNA fragment, which is produced by a specific cut in the 28-36-nucleotide (nt) anticodon ring and is a mature tRNA, known as tRNA-derived stress-induced RNA or tiRNA; the other tsRNA is 14-30 nt in length and known as a tRNA-derived fragment (tRF) (11). tRFs/tRNA-derived RNAs (tDRs) are derived from mature tRNA or precursor tRNA, which can be subdivided as follows: i) tRF-5', corresponding to the 5' end of the mature tRNA according to its corresponding position on the tRNA, obtained by D-loop cleavage; ii) tRF-3', corresponding to the 3 ' end of the mature tRNA and containing a CCA tail sequence, obtained by T-loop cleavage; and iii) tRF-1, which is the 3'-tail sequence of the precursor tRNA and contains a poly $\mathrm{T}$ sequence at the 3 ' end (12-14).

tsRNA is not only a by-product of random tRNA cleavage but also a small noncoding RNA that has crucial roles in numerous specific physiological and pathological processes (11). Additionally, tsRNA has an impact on the 
function of various types of cells. Studies have demonstrated that tsRNAs are present in a variety of organisms and affect different biological processes, such as the growth and metastasis of cancer cells, tumor inhibition, regulation of gene and protein expression, initiation of viral reverse transcriptase, RNA processing, the DNA damage response and neurodegeneration (15-18). However, the role of tsRNAs in hypertrophic scar fibroblasts has not yet been reported.

Therefore, the present study investigated differentially expressed tsRNAs in human hypertrophic scar fibroblasts and normal skin fibroblasts via high-throughput sequencing. tsRNAs with partial differential expression were confirmed by reverse transcription-quantitative polymerase chain reaction (RT-qPCR). Bioinformatics techniques such as target gene prediction, Gene Ontology (GO) and Kyoto Encyclopedia of Genes and Genomes (KEGG) pathway enrichment were then used to investigate the role of tsRNA differential expression. Some of the differentially expressed tsRNAs were selected to generate coexpression and competing endogenous RNA (ceRNA) networks. The main purpose of the study was to elucidate the potential molecular mechanism of tsRNAs in proliferative scars and to lay a foundation for the treatment of such scars.

\section{Materials and methods}

Preparation of fibroblasts. The present study was approved by the Ethics Committee of the First Affiliated Hospital of Nanchang University (Nanchang, China). Written informed consent was obtained from the adult participants and from the parent of the child participant. Hypertrophic scars and adjacent normal skin tissues were harvested from three female patients (aged 6, 20 and 26 years) who were admitted to the First Affiliated Hospital of Nanchang University from April to October 2018. A typical manifestation of hypertrophic scar is a bulging, erythematous, itching and thickened scar restricted to the site of injury $(19,20)$. Samples were obtained from patients with hypertrophic scars following perineal burns that had not been treated with drugs or radiotherapy prior to sample collection. The samples were washed three times with PBS, and the subcutaneous tissue was removed. The tissue was placed in a Petri dish and digested with $0.25 \%$ trypsin-EDTA (Gibco; Thermo Fisher Scientific, Inc.) at $4^{\circ} \mathrm{C}$ for $10-12 \mathrm{~h}$. The dermal layer of the tissue was retained; the tissue epidermis was completely removed to avoid contamination of the epidermal cells, and the dermis was cut into $1 \times 1-\mathrm{mm}$ pieces with ophthalmic scissors (21). The cut dermal tissue pieces were plated in a Petri dish, and DMEM containing $10 \%$ fetal calf serum (Gibco; Thermo Fisher Scientific, Inc.) was added. The cells were incubated at $37^{\circ} \mathrm{C}$ under $5 \% \mathrm{CO}_{2}$, and the medium was changed every 3 days. The cells disintegrated, expanded and fused into sheets covering $80 \%$ of the bottom of the culture dish; they were then digested and passaged. Fibroblasts of the third generation were used in the study. The fibroblasts derived from hypertrophic scar tissue were designated as the experimental group, and those derived from normal skin were designated as the control group.

RNA isolation and quality control. Total RNA was extracted using TRIzol reagent according to the manufacturer's protocol
(Invitrogen; Thermo Fisher Scientific, Inc.). The quantity of extracted RNA was measured using a NanoDrop ONE spectrophotometer (Thermo Fisher Scientific, Inc.), and the quality of the extracted RNA, specifically, its integrity and purity, was assessed by standard denaturing agarose gel electrophoresis. The optical density ratio at wavelengths of 260 and $280 \mathrm{~nm}$ (OD260/280) was measured as an indicator of RNA concentration and was used to determine RNA purity; an OD260/280 ratio of between 1.8 and 2.1 is considered to indicate acceptable RNA quality $(22,23)$.

Library preparation for RNA sequencing ( $R N A-s e q)$. The library was created by first adding a $3^{\prime}$ linker sequence and sequentially adding $1 \mu \mathrm{l}$ SR RT Primer, $4.5 \mu \mathrm{l}$ nuclease-free $\mathrm{H}_{2} \mathrm{O}$ for reverse transcription primer hybridization, and the $5^{\prime}$ adaptor sequence. RNA fragments were used to synthesize cDNA strands as follows: Degeneration at $94^{\circ} \mathrm{C}$ for $15 \mathrm{sec}$, annealing at $62^{\circ} \mathrm{C}$ for $30 \mathrm{sec}$, and extension at $70^{\circ} \mathrm{C}$ for $15 \mathrm{sec}$. The library was then examined on an $8 \%$ SDS-PAGE Tris-borate-EDTA gel after $1 \mathrm{~h}$ at $120 \mathrm{~V}$. The library was constructed using a HiSeq X Reagent kit v2.5 (FC-501-2501; Illumina, Inc.). Library quality was assessed using an Agilent 2200 TapeStation system (Agilent Technologies, Inc.) prior to sequencing. Total concentrations were determined using a Qubit 3.0 Fluorometer (Thermo Fisher Scientific, Inc.). Libraries were sequenced using a HiSeq X Ten high-throughput sequencer (Illumina, Inc.) with $2 \times 150$-bp paired-end reads. Purified cDNA libraries were used for cluster generation (cBot Cluster Generator; Illumina, Inc.). The library was sequenced and constructed by Shanghai Personal Biotechnology Co., Ltd. (www.personalbio.cn/).

Data quality control. First, tsRNA sequences that did not match sequences in miRBase (http://www.mirbase.org) were matched to sequences in the GtRNA database version 18.1 (24). The tsRNA sequences that matched to the GtRNA database were then searched against the tRFdb 2012 (25) and tRF MINTbase v2.0 (26) databases. The expressed tsRNAs in the tissue were identified. In the miRNA analysis, the filtered clean reads were compared with the human miRNA database 22.1 (http://www.mirbase.org/) to obtain miRNA expression data. Levels of tsRNA and miRNA gene expression were obtained by RNA-seq. The level of gene expression is closely associated with the following two aspects: i) The level of gene expression and the number of reads in the mapping reference sequence, that is, the effective sequencing amount; ii) the sum of the length of all exons of the gene, as increased gene transcription generates more sequencing fragment results (27). The number of reads of each gene was then obtained. According to the known small RNA data, the differences in small RNAs between the hypertrophic scar fibroblasts and normal skin fibroblasts were determined. Differentially expressed genes were obtained using DEGseq Software (version 1.18.0) (28). The selection criteria were fold change $>2.0, \mathrm{P}<0.05$ and false discovery rate $(\mathrm{FDR})<0.05$.

Target prediction. TargetScan software (www.targetscan.org/) was used to predict the potential binding targets of mRNAs, and target genes were predicted by searching for conserved 8- and 7-mer sites that matched each miRNA seed region. 
Table I. Primer sequences of tsRNAs used for reverse transcription-quantitative PCR.

\begin{tabular}{|c|c|c|c|}
\hline Primer ID & Primer sequence $\left(5^{\prime}-3^{\prime}\right)$ & Length (bp) & Annealing temperature $\left({ }^{\circ} \mathrm{C}\right)$ \\
\hline \multirow[t]{2}{*}{ U6 } & F: CGATACAGAGAAGATTAGCATGGC & \multirow[t]{2}{*}{61} & \multirow[t]{2}{*}{60} \\
\hline & R: AACGCTTCACGAATTTGCGT & & \\
\hline \multirow[t]{3}{*}{ tsRNA-23678 } & F: GGGGGTATAGCTCAGGGGTAGA & \multirow[t]{3}{*}{72} & \multirow[t]{3}{*}{60} \\
\hline & R: GTCGTATCCAGTGCGTGTCGTGGAGTCGGCAA & & \\
\hline & TTGCACTGGATACGACAGTCAAA & & \\
\hline \multirow[t]{3}{*}{ tsRNA-23727 } & F: GGGGGTATAGCTCAGTGGTAGAG & \multirow[t]{3}{*}{71} & \multirow[t]{3}{*}{60} \\
\hline & R: GTCGTATCCAGTGCGTGTCGTGGAGTCGGCAA & & \\
\hline & TTGCACTGGATACGACAGTCAAA & & \\
\hline \multirow[t]{3}{*}{ tsRNA-23761 } & F: GGGGGTGTAGCTCAGTGGTAGAG & \multirow[t]{3}{*}{71} & \multirow[t]{3}{*}{60} \\
\hline & R: GTCGTATCCAGTGCGTGTCGTGGAGTCGGCAA & & \\
\hline & TTGCACTGGATACGACAGTCAAA & & \\
\hline
\end{tabular}

tsRNA, tRNA-derived small RNA; F, forward; R, reverse.

The miRanda (http://www.microrna.org) and RNAhybrid (https://bibiserv.cebitec.uni-bielefeld.de/) algorithms were employed to predict mRNAs targeted by differentially expressed tsRNAs (screening criteria: miRanda, score $\geq 150$ and energy <-20; RNAhybrid, energy <-25). The intersection of the two algorithms was taken as the final result of the target gene prediction. The miRanda score is a binding score of the tsRNA and its target gene, with a higher score indicating a more accurate target gene prediction.

GO analysis. The predicted target genes were subjected to GO analysis (www.geneontology.org/), and all involved GO genes were evaluated. The significance level (P-value) of each GO gene was calculated by Fisher's test, the results were corrected using the FDR method of multiple hypothesis testing to identify significant GO genes from differential gene enrichment at $\mathrm{P}<0.05$. The $\mathrm{P}$-values for the enrichment of $\mathrm{GO}$ terms were calculated by the default statistical algorithm of the GO analysis database. A smaller P-value indicates a more significant role of a GO term, and terms with $\mathrm{P}<0.05$ were considered statistically significant. Under the same P-value, a larger enrichment score indicates a more specific effect of a gene.

Pathway analysis. KEGG pathway (www.kegg.jp/) enrichment analysis of the differentially expressed tsRNAs was performed. All pathways in which the genes are involved were determined based on the hypergeometric distribution of Fisher's test calculation of each pathway significance level (P-value), the results of multiple hypothesis correction tests and the FDR. Thus, significant pathways embodied by differential genes were identified. The standard for significance screening was $\mathrm{P}<0.05$; biological pathways with $\mathrm{P}<0.05$ were considered significant.

Validation by RT-qPCR. To confirm the RNA-seq data, three tsRNAs were selected for verification by RT-qPCR. First, cDNA was synthesized using an ABI Q6 RT-qPCR system (Applied Biosystems; Thermo Fisher Scientific, Inc.). RT-qPCR was performed in a $12-\mu 1$ reaction mixture containing $1 \mathrm{ng}$ total RNA, $1 \mu \mathrm{l}$ RT primer and $2 \mu \mathrm{ldNTP}$ mix (HyTest Ltd.),
$4 \mu \mathrm{l}$ X buffer, $1 \mu \mathrm{l}$ Protector RNase Inhibitor and $1 \mu \mathrm{l}$ transcriptase (all from Epicentre; Illumina Inc.). The stem-loop RT reaction was performed at $65^{\circ} \mathrm{C}$ for $5 \mathrm{~min}$, followed by $42^{\circ} \mathrm{C}$ for $60 \mathrm{~min}$ and $70^{\circ} \mathrm{C}$ for $5 \mathrm{~min}$. RT-qPCR was performed with the 2X FastStart Universal SYBR-Green Master mix kit (Roche) with the following amplification procedure: $95^{\circ} \mathrm{C}$ for $10 \mathrm{~min}$, followed by 45 cycles of $95^{\circ} \mathrm{C}$ for $15 \mathrm{sec}$ and $60^{\circ} \mathrm{C}$ for $60 \mathrm{sec}$ for fluorescence collection. After the amplification reaction was complete, a melting curve of the RT-qPCR product was established during fluorescence collection $\left(95^{\circ} \mathrm{C}\right.$ for $10 \mathrm{sec}$; $60^{\circ} \mathrm{C}$ for $60 \mathrm{sec}$; and $95^{\circ} \mathrm{C}$ for $15 \mathrm{sec}$ ) by slowly heating from 60 to $99^{\circ} \mathrm{C}\left(+0.05^{\circ} \mathrm{C} / \mathrm{sec}\right)$. The target gene and internal reference gene of each sample were subjected to RT-qPCR. Primers were synthesized by Shanghai Bioligo Biotechnology Co., Ltd., as listed in Table I. The U6 gene was used as an internal reference for primer design. RT-qPCR quantification was performed using the $2^{-\Delta \Delta \mathrm{Cq}}$ method (29).

Expression of several tsRNAs and miRNAs in hypertrophic scar tissue and normal skin tissue. Detection of tsRNA-23678, tsRNA-23761, tsRNA-23727, miR 29b-1-5p and miR-3135b gene expression was performed using RT-qPCR. The hypertrophic scar and normal skin tissue were cut into small pieces and ground into fine powder in liquid nitrogen, using a mortar and pestle. Total RNA was extracted using TRIzol reagent. The Rayscript cDNA Synthesis kit (cat. no. GK8030; Shanghai Generay Biotech Co., Ltd.) was used to synthesize cDNA via reverse transcription using the following temperature protocol: $95^{\circ} \mathrm{C}$ for $10 \mathrm{~min}$, followed by 45 cycles of $95^{\circ} \mathrm{C}$ for $15 \mathrm{sec}$ and $60^{\circ} \mathrm{C}$ for $60 \mathrm{sec}$ for fluorescence collection. The specific cDNA fragments were amplified using a RT-qPCR detection system and primers from Shanghai Bioligo Biotechnology Co., Ltd., as described above. Data are expressed as expression relative to the U6 gene. All reactions were analyzed using the $2^{-\Delta \Delta \mathrm{Cq}}$ method.

Statistical analysis. Differences in tsRNA levels between hypertrophic scars and adjacent normal skin samples were analysed using a paired Student's t-test The FDR was calculated to correct the P-values. Differential expression of 
tsRNAs was defined by a fold-change threshold of $>2.0$ and $\mathrm{P}<0.05$. $\mathrm{P}<0.05$ was considered to indicate statistical significance. Statistical analyses were performed using SPSS version 19 software (IBM Corp).

tSRNA-miRNA coexpression network and ceRNA network analyses. The differentially expressed tsRNAs contain miRNA binding sites. Three differentially expressed tsRNAs were selected and networks containing them were established. High-throughput sequencing results indicated that tsRNA-23678, tsRNA-23727 and tsRNA-23761 were upregulated in the hypertrophic scar fibroblasts. The tsRNAs and miRNAs associated with the same target gene were obtained. The miRNAs with the same expression trends as the tsRNAs were identified, and significant target genes were screened on the basis of their $\mathrm{P}$-values $(\mathrm{P}<0.05)$. First, Cytoscape 3.6.1 (www.cytoscape.org/) was used to construct a tsRNA-miRNA coexpression network and a ceRNA network. The purpose of constructing these networks was to discover potential miRNA reaction elements. The overlap of a binding site for both the tsRNA and miRNA on the same mRNA may aid in the prediction of tsRNA-miRNA-mRNA interactions. The intersections of results from the commonly used software miRanda, Targetscan and psRobot (omicslab.genetics.ac.cn/psRobot/) were used to predict tsRNA-miRNA-mRNA interactions.

\section{Results}

Extraction of total RNA. Denaturing gel electrophoresis revealed intense and distinct $28 \mathrm{~S}$ and $18 \mathrm{~S}$ ribosomal RNA bands. A smaller, slightly diffuse band consisting of low molecular weight RNA (tRNA and 5S ribosomal RNA) was also observed (Fig. S1). As shown in Table II, the extracted RNAs were acceptable for subsequent tsRNA experiments.

Differential tsRNA and miRNA expression profiles. High-throughput sequencing is an efficient method for studying the biological function of RNAs. tsRNA expression profiles in fibroblasts were obtained by RNA-seq. Thousands of transcripts were detected in the hypertrophic scars and normal tissues by RNA-seq. Among them, 67 differentially expressed tsRNAs were detected (Tables III and IV), of which 27 were upregulated and 40 downregulated in hypertrophic scar fibroblasts. The heatmap of intersample association (Fig. 1) showed an evident difference in transcript levels between hypertrophic scar and normal skin fibroblasts. A total of 149 miRNAs were significantly differentially expressed (fold change $>2.0$, $\mathrm{P}<0.05)$. Of these, $120 \mathrm{miRNAs}$ were upregulated and 29 downregulated. The top 15 upregulated and top 15 downregulated significantly differentially expressed miRNAs are shown Table V. These results reveal that the expression profiles of tsRNAs and miRNAs in hypertrophic scar fibroblasts differed from those in normal skin fibroblasts.

$G O$ analysis. To explore the potential functions of tsRNAs in hypertrophic scars, $\mathrm{GO}$ analysis of differentially expressed tsRNAs was performed. The important GO terms and the genes involved were obtained via the gene function analysis of target genes. Fig. 2 shows the degree of enrichment of the top 15 genes identified, with the enrichment degree listed in descending
Table II. RNA quantification and quality assurance by spectrophotometry.

\begin{tabular}{lcccc}
\hline Group & $\begin{array}{c}\text { OD260/280 } \\
\text { ratio }\end{array}$ & $\begin{array}{c}\text { Concentration } \\
(\mathrm{ng} / \mu \mathrm{l})\end{array}$ & $\begin{array}{c}\text { Quantity } \\
(\mathrm{ng})\end{array}$ & Result \\
\hline HA & 2.10 & 653.4 & 13.07 & Qualified \\
HB & 2.05 & 681.6 & 13.63 & Qualified \\
\hline
\end{tabular}

OD260/280, ratio of optical density at 260 and $280 \mathrm{~nm}$; HA, hypertrophic scar; HB, normal skin.

order. In the $-\log _{10}$ calculation of the P-value, a smaller P-value corresponds to a greater $-\log _{10} \mathrm{P}$-value. The GO project covers three main domains, namely biological processes (BP), molecular function (MF) and cellular component (CC). In total, 5,891 genes showed differences in the GO analysis. The data indicate that genes in the BP category are mainly associated with the following: 'Homophilic cell adhesion' via plasma membrane adhesion molecules; 'cell adhesion'; 'positive regulation of transcription,' DNA-templated; 'epidermal growth factor receptor'; and 'negative regulation of cell migration'. Genes in the MF category are mainly associated with 'metal ion binding', 'protein binding' and 'calcium ion binding'; genes in the CC category are mainly associated with 'cytoplasm', 'cell junction', 'plasma membrane' and 'cytosol'. In summary, these data indicate that differentially expressed target gene tsRNAs may be involved in cell division and proliferation.

KEGG pathway analysis. KEGG pathway analysis indicated that the dysregulated tsRNAs are involved in numerous signaling pathways, with 283 KEGG pathways associated with the differentially expressed tsRNAs. A histogram showing the $-\log _{10} \mathrm{P}$-values of KEGG pathways is shown in Fig. 3. The - $\log _{10}$ (P-value) denotes an enrichment score for the significance of pathway correlations. The histogram shows the top 15 enriched pathways, and the most significantly altered pathways included the 'Ras signaling pathway', 'Rap1 signaling pathway' and the 'cGMP-PKG signaling pathway'. These genes may serve important roles in cell proliferation, differentiation, metastasis and apoptosis.

$m R N A$-target prediction of tsRNA. The results showed that one tsRNA can target multiple mRNAs and that one mRNA can also be a target gene for multiple tsRNAs. As shown in Table VI, the target genes of tsRNA-23678 were predicted to be FAS, SMAD2, ABCA1, ABCCB, ABHDS, ABL2, AIPL1 and BB59. Predicted target genes of tsRNA-23761 were COL1A, A1CF, $\mathrm{ABI} 3, \mathrm{BEST} 1, \mathrm{ABCA} 1, \mathrm{ACAP} 3, \mathrm{ACE}$ and B3GNT7, and those of tsRNA-23727 were SMAD2, TGFBR1, ABCC8, ABHD5, ABL2, BEAN1, AIPH and BAALC. SMAD2 was a target gene for both tsRNA-23678 and tsRNA-23727. In the target gene prediction, the miRanda scores of tsRNA23727, tsRNA23761 and tsRNA23678 were 165,150 and 157, respectively.

Validation with RT-qPCR. The differentially expressed tsRNA-23678, tsRNA-23727 and tsRNA-23761 were chosen for further analysis to verify the RNA-seq results. Amplification and dissociation curves for these tsRNAs were 
Table III. Data for significant differentially expressed upregulated tsRNAs of hypertrophic scar compared with normal skin.

\begin{tabular}{|c|c|c|c|c|c|}
\hline Yingbio ID & Fold change & Database ID & $\log _{2} \mathrm{FC}$ & Anticodons & Type \\
\hline tsRNA-04990 & 4.2733 & tRF-33-LQR47673FEWSD3 & 2.0953 & GlyGCC & 5'-half \\
\hline tsRNA-04998 & 2.0229 & tRF-33-LQ947673FEWSD3 & 1.0164 & GlyCCC & 5'-half \\
\hline tsRNA-06150 & 2.0243 & tRF-33-FQ947673FEWSD3 & 1.0174 & GlyCCC & 5'-half \\
\hline tsRNA-14697 & 2.0476 & tRF-31-P4R8YP9LON4VD & 1.0339 & GlyCCC & 5'-half \\
\hline tsRNA-14698 & 4.1770 & tRF-32-P4R8YP9LON4V3 & 2.0624 & GlyCCC & 5'-half \\
\hline tsRNA-14699 & 4.1187 & tRF-33-P4R8YP9LON4VDP & 2.0421 & GlyCCC & 5'-half \\
\hline tsRNA-14700 & 4.0846 & tRF-34-P4R8YP9LON4VHM & 2.0302 & GlyCCC & 5'-half \\
\hline tsRNA-14701 & 4.1227 & tRF-35-P4R8YP9LON4VN1 & 2.0435 & GlyCCC & tRF-5 \\
\hline tsRNA-14702 & 4.0472 & tRF-36-P4R8YP9LON4VN1B & 2.0169 & GlyCCC & tRF-5 \\
\hline tsRNA-14703 & 3.9967 & tRF-38-P4R8YP9LON4VN18 & 1.9988 & GlyCCC & tRF-5 \\
\hline tsRNA-14704 & 3.9166 & tRF-40-P4R8YP9LON4VN1EH & 1.9696 & GlyCCC & tRF-5 \\
\hline tsRNA-14705 & 3.9591 & tRF-43-P4R8YP9LON4VN1EH & 1.9851 & GlyCCC & tRF-5 \\
\hline tsRNA-14771 & 2.0016 & tRF-37-PNR8YP9LON4V4R1 & 1.0012 & GlyCCC & tRF-5 \\
\hline tsRNA-23678 & 5.2361 & tRF-32-RKVP4PRL5FZU3 & 2.3885 & CysGCA & 5'-half \\
\hline tsRNA-23679 & 4.3826 & tRF-33-RKVP4PRL5FZUDP & 2.1317 & CysGCA & 5'-half \\
\hline tsRNA-23680 & 3.9089 & tRF-34-RKVP4PRL5FZUHM & 1.9667 & CysGCA & 5'-half \\
\hline tsRNA-23727 & 8.8052 & tRF-32-RKVP4P9L5FZU3 & 3.1383 & CysGCA & 5'-half \\
\hline tsRNA-23728 & 7.1609 & tRF-33-RKVP4P9L5FZUDP & 2.8401 & CysGCA & 5'-half \\
\hline tsRNA-23729 & 7.3594 & tRF-33-RKVP4P9L5FZUDP & 2.8795 & CysGCA & 5'-half \\
\hline tsRNA-23730 & 7.1105 & tRF-34-RKVP4P9L5FZUHM & 2.8299 & CysGCA & tRF-5 \\
\hline tsRNA-23761 & 5.9145 & tRF-35-RKVP4P9L5FZUNF & 2.5642 & CysGCA & 5'-half \\
\hline tsRNA-23762 & 4.3390 & tRF-32-RK9P4P9L5FZU3 & 2.1173 & CysGCA & 5'-half \\
\hline tsRNA-23772 & 3.4717 & tRF-33-RK9P4P9L5FZUDP & 1.7956 & AlaCGC & 5'-half \\
\hline tsRNA-23774 & 3.5778 & tRF-31-RK9P4P9L5HMVE & 1.8391 & AlaCGC & 5'-half \\
\hline tsRNA-23775 & 3.9072 & tRF-32-RK9P4P9L5HMVQ & 1.9661 & AlaCGC & 5'-half \\
\hline tsRNA-26551 & 3.3115 & tRF-33-RK9P4P9L5HMV05 & 1.7274 & SerGCT & tRF-1 \\
\hline tsRNA-26578 & 2.1116 & tsRNA-1005 & 1.0783 & GlyTCC & tRF-1 \\
\hline
\end{tabular}

tsRNA, tRNA-derived small RNA; database ID, from the tRFdb database 2012; tRF, tRNA-derived fragment.

generated (Fig. 4), and the $2{ }^{\Delta \Delta \mathrm{Cq}}$ values of the tsRNAs were calculated according to the relative quantitative method. The $2^{-\Delta \Delta \mathrm{Cq}}$ analysis revealed upregulation of tsRNA-23678 (1.9452-fold), tsRNA-23727 (1.6684-fold) and tsRNA-23761 (1.6997-fold) in hypertrophic scar, which is consistent with the RNA-seq results (Fig. 5; Table VII).

Expression of the several tsRNAs and miRNAs between hypertrophic scar tissue and normal skin tissue. In order to verify the aforementioned results, RT-qPCR assays were performed on selected tsRNAs (tsRNA-23678, tsRNA-23727 and tsRNA-23761) and miRNAs (miR-3135b and miR-29b-1-5p). Compared with normal skin tissue, the expression levels of tsRNA-23678, tsRNA-23727, tsRNA-23761 and miR-3135b were significantly upregulated in hypertrophic scar tissue, whereas the expression of miR-29b-1-5p was significantly downregulated in hypertrophic scar tissue (Fig. 6). These results demonstrate that the changes observed in tissue were consistent with the bioinformatics results.

Construction of coexpression networks. The functions of most tsRNAs are not currently annotated. The functional prediction of tsRNAs is based on the annotation of coexpressed miRNAs, and three differentially expressed tsRNAs in fibroblasts were chosen in the present study according to the degree of correlation. The coexpression network (Fig. 7) showed that one tsRNA might be associated with one or more miRNAs. A total of 40 miRNAs were associated with the three tsRNAs. Furthermore, the coexpression networks indicated these tsRNAs to be involved in a number of biological processes, including cell adhesion, proliferation, differentiation and metastasis. The network analysis also demonstrated that tsRNA-23678 is associated with miR-29b-1-5p, miR-222-3p and miR-423-5p, which had the same trends in expression. This finding aids in the identification of regulatory relationships between tsRNAs and miRNAs in hypertrophic scars.

Construction of ceRNA networks. The ceRNA network hypothesis provides a new mechanism for tsRNA-miRNA-mRNA interactions. miRNAs are known to cause gene silencing by binding to mRNA, and tsRNAs may regulate gene expression by competitively binding to miRNAs. Thus, tsRNAs can be considered as ceRNAs. According to the ceRNA hypothesis, numerous non-coding RNAs may function as ceRNAs, which 
Table IV. Data for significant differentially expressed downregulated tsRNAs of hypertrophic scar compared with normal skin.

\begin{tabular}{|c|c|c|c|c|}
\hline Yingbio_ID & Fold change & Database_ID & $\log _{2} \mathrm{FC}$ & Anticodons \\
\hline tsRNA-05068 & 0.3915 & tRF-34-LSXMSL73VL4YHE & -1.3527 & HisGTG \\
\hline tsRNA-05069 & 0.2660 & tRF-34-LSXMSL73VL4YHE & -1.9100 & HisGTG \\
\hline tsRNA-05070 & 0.2673 & tRF-35-LSXMSL73VL4YMY & -1.9033 & HisGTG \\
\hline tsRNA-05071 & 0.2711 & tRF-36-LSXMSL73VL4YMYE & -1.8828 & HisGTG \\
\hline tsRNA-06211 & 0.3743 & tRF-33-FSXMSL73VL4YDN & -1.4174 & HisGTG \\
\hline tsRNA-06212 & 0.2695 & tRF-34-FSXMSL73VL4YHE & -1.8916 & HisGTG \\
\hline tsRNA-06213 & 0.2575 & tRF-35-FSXMSL73VL4YMY & -1.9569 & HisGTG \\
\hline tsRNA-06214 & 0.2562 & tRF-36-FSXMSL73VL4YMYE & -1.9642 & HisGTG \\
\hline tsRNA-10278 & 0.3460 & tRF-32-6SXMSL73VL4YK & -1.5309 & HisGTG \\
\hline tsRNA-10279 & 0.3335 & tRF-33-6SXMSL73VL4YDN & -1.5840 & HisGTG \\
\hline tsRNA-10280 & 0.2544 & tRF-34-6SXMSL73VL4YHE & -1.9747 & HisGTG \\
\hline tsRNA-10281 & 0.2471 & tRF-35-6SXMSL73VL4YMY & -2.0162 & HisGTG \\
\hline tsRNA-10282 & 0.2668 & tRF-36-6SXMSL73VL4YMYE & -1.9057 & HisGTG \\
\hline tsRNA-10283 & 0.2766 & tRF-37-6SXMSL73VL4YMYP & -1.8538 & HisGTG \\
\hline tsRNA-15103 & 0.3519 & tRF-31-PW5SVP9N15WV0 & -1.5064 & HisGTG \\
\hline tsRNA-15104 & 0.3098 & tRF-32-PW5SVP9N15WVN & -1.6902 & HisGTG \\
\hline tsRNA-15105 & 0.2369 & tRF-33-PW5SVP9N15WV0E & -2.0770 & HisGTG \\
\hline tsRNA-15106 & 0.2555 & tRF-34-PW5SVP9N15WV2P & -1.9682 & HisGTG \\
\hline tsRNA-15107 & 0.2545 & tRF-35-PW5SVP9N15WV7W & -1.9739 & HisGTG \\
\hline tsRNA-15108 & 0.2731 & tRF-36-PW5SVP9N15WV7W0 & -1.8720 & HisGTG \\
\hline tsRNA-15109 & 0.2728 & tRF-39-PW5SVP9N15WV7WIV & -1.8737 & HisGTG \\
\hline tsRNA-15110 & 0.2806 & tRF-40-PW5SVP9N15WV7W6S & -1.8331 & HisGTG \\
\hline tsRNA-15111 & 0.2966 & tRF-45-PW5SVP9N15WV7W6SJF & -1.7533 & HisGTG \\
\hline tsRNA-17436 & 0.3188 & tRF-34-94U47P299DZUFJ & -1.6489 & GlnTTG \\
\hline tsRNA-18787 & 0.2153 & tRF-35-VHJYOZ4E8BRY93 & -2.2149 & ArgTCT \\
\hline tsRNA-19074 & 0.4575 & tRF-37-V4QP596VZ631QJJ & -1.1279 & GluTTC \\
\hline tsRNA-21604 & 0.3724 & tRF-32-XSXMSL73VL4YK & -1.4247 & HisGTG \\
\hline tsRNA-21605 & 0.2994 & tRF-33-XSXMSL73VL4YDN & -1.7395 & HisGTG \\
\hline tsRNA-21606 & 0.2578 & tRF-34-XSXMSL73VL4YHE & -1.9551 & HisGTG \\
\hline tsRNA-21607 & 0.2407 & tRF-35-XSXMSL73VL4YMY & -2.0546 & HisGTG \\
\hline tsRNA-21608 & 0.2578 & tRF-36-XSXMSL73VL4YMYE & -1.9551 & HisGTG \\
\hline tsRNA-21609 & 0.2653 & tRF-37-XSXMSL73VL4YMYP & -1.9141 & HisGTG \\
\hline tsRNA-21610 & 0.2803 & tRF-40-XSXMSL73VL4YMY91 & -1.8348 & HisGTG \\
\hline tsRNA-21611 & 0.2802 & tRF-42-XSXMSL73VL4YMY91M & -1.8350 & HisGTG \\
\hline tsRNA-24180 & 0.3091 & tRF-32-ROD8N0X0JYOYO & -1.6937 & TyrGTA \\
\hline tsRNA-24181 & 0.2991 & tRF-33-ROD8N0X0JYOYOF & -1.7411 & TyrGTA \\
\hline tsRNA-24182 & 0.2995 & tRF-34-ROD8N0X0JYOY26 & -1.7389 & TyrGTA \\
\hline tsRNA-24183 & 0.3123 & tRF-36-ROD8N0X0JYOYUED & -1.6787 & TyrGTA \\
\hline tsRNA-24184 & 0.3030 & tRF-37-ROD8N0X0JYOYUE3 & -1.7222 & TyrGTA \\
\hline tsRNA-26579 & 0.4958 & tsRNA-1042 & -1.0121 & ThrAGT \\
\hline
\end{tabular}

tsRNA, tRNA-derived small RNA; database ID, from the tRFdb database 2012; tRF, tRNA-derived fragment.

compete for the same microRNA response elements (MREs) and regulate each other (21). The analysis of ceRNA interactions aids in the functional characterization of such noncoding transcripts. A tsRNA-miRNA-mRNA network associated with hypertrophic scars was established in the present study using high-throughput sequencing data (Fig. 8). In this network, tsRNA-23761 was positively associated with miR-3135b. Furthermore, the network indicates that tsRNA-23761 is a
ceRNA of miR-3135b that targets angiotensin-converting enzyme (ACE) and PYGO2, and tsRNA-23678 is a ceRNA of miR-133a-3p that targets FXR2 and PNAGS.

\section{Discussion}

tsRNA, which is derived from tRNA, is a newly discovered class of small molecular RNAs that are produced by the cleavage of 


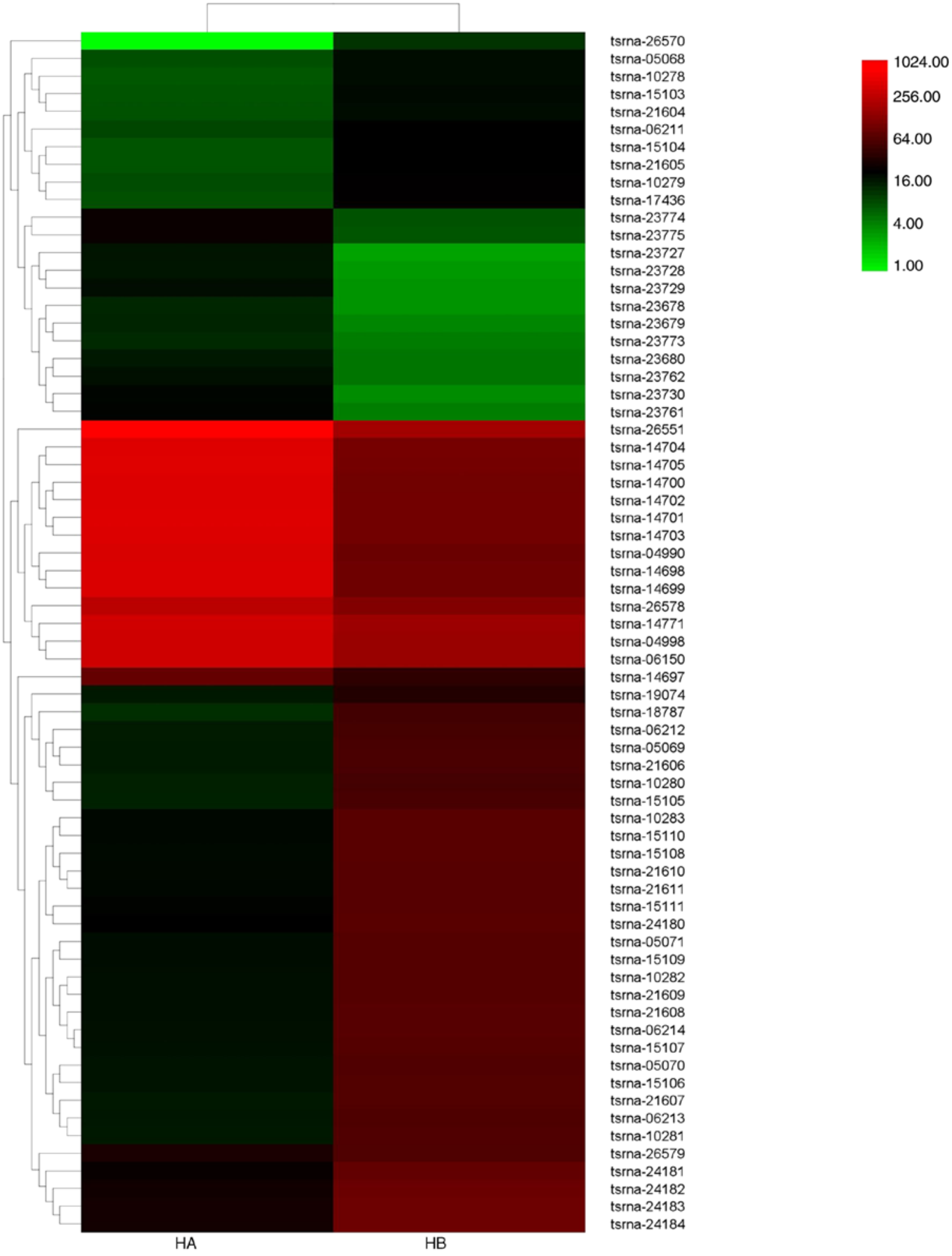

Figure 1. Heatmap showing expression profiles of differentially expressed tsRNAs. The map is based on the expression values of all expressed tsRNAs detected by RNA sequencing with fold change $>2.0, \mathrm{P}<0.05$ and a false discovery rate $<0.05$. The expression values are indicated by the color scale; red indicates high relative expression and green indicates low relative expression. tsRNA, tRNA-derived small RNA.

the tRNA ring by Dicer or angiogenin enzymes $(11,30)$. tsRNA can be classified into five different types: 5'- and 3'-tRNA fragments (tRFs); 5'-and 3'-halves; and 3'U tRFs (31,32). There is growing evidence that that tsRNAs are associated with the development of tumors, cell proliferation and viral replication (16), regulation of cell viability (33), inhibition of protein translation $(34,35)$, regulation of cancer progression (36), offspring metabolism $(37,38)$ and numerous other processes. It has also been reported that tsRNA may have a regulatory function similar to that of miRNA, which can act like a sponge to regulate mRNA stability and participate in gene transcription and translation (33). However, the role of tsRNA in hypertrophic scars has not yet been reported. Hypertrophic scar is a fibrotic disorder, mainly due to the response of the body to 
A

GO-Analysis_BP

-Log10 (P-value)

$$
\begin{array}{lllllllllll}
0 & 1 & 2 & 3 & 4 & 5 & 6 & 7 & 8 & 9 & 10
\end{array}
$$

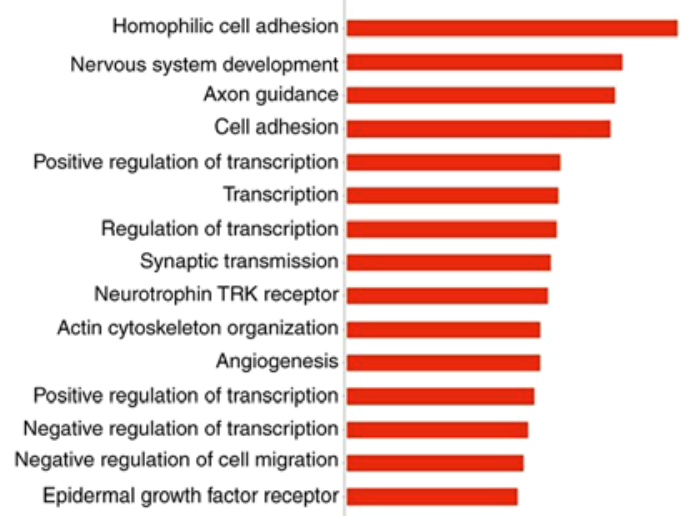

B

GO-Analysis_MF

$-\log 10(P$-value $)$

$\begin{array}{llllllllllll}0 & 1 & 2 & 3 & 4 & 5 & 6 & 7 & 8 & 9 & 10 & 11\end{array}$

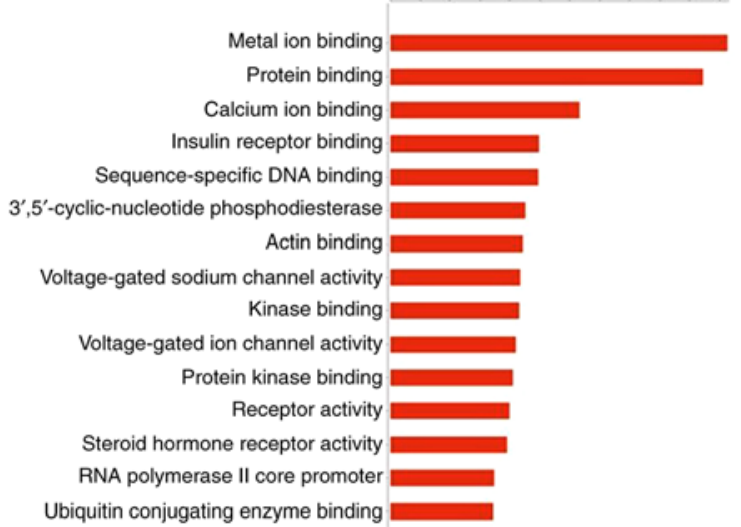

C

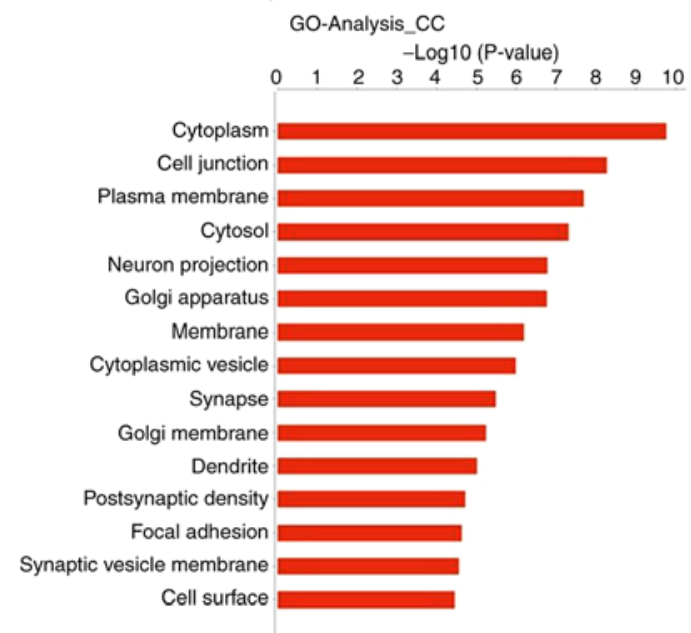

Figure 2. GO analysis of differentially expressed tsRNAs. The top 15 enriched terms covering (A) BP, (B) MF and (C) CC are presented. GO, Gene Ontology; tsRNAs, tRNA-derived small RNA. BP, biological processes; $\mathrm{MF}$, molecular functions; $\mathrm{CC}$, cellular components.

injury, and caused by the excessive proliferation of fibroblasts and excessive production of ECM (39). Understanding the relationship between hypertrophic scars and tsRNA may help to elucidate the pathogenesis and pathophysiology of hypertrophic scars and identify potential therapeutic targets for their clinical treatment.

In the present study, high-throughput sequencing was first applied to evaluate the differentially expressed profiles of
tsRNAs and miRNAs between hypertrophic scar fibroblasts and normal skin fibroblasts. Differentially expressed tsRNAs and miRNAs were identified, including 27 upregulated tsRNAs, such as tsRNA-23678, tsRNA-23727, tsRNA-23761, tsRNA-04998, tsRNA-06150 and tsRNA-14697, and 40 downregulated tsRNAs, such as tsRNA-05068, tsRNA-05070, tsRNA-06213, tsRNA-06214, tsRNA-10278 and tsRNA-10279. In addition, 120 upregulated miRNAs, including miR-3135b, miR-10a-5p, miR-10b-5p, miR-129-5p and miR-133a-3p, and 29 downregulated miRNAs, including miR-29b-1-5p, miR-1275, miR-148b-5p, miR-151b and miR-18a-5p were identified. Furthermore, GO functional enrichment and KEGG pathway enrichment analyses were performed. These differentially expressed tsRNAs were found to be enriched in a number of important biological processes, including 'nervous system development', 'cell adhesion', 'focal adhesion', 'protein binding', 'angiogenesis' and 'actin binding'. Some biological signaling pathways, such as the 'Ras signaling pathway', the 'Rap1 signaling pathway' and the 'cGMP-PKG signaling pathway' were also significantly enriched. These pathways are associated with the formation of new tissue, remodeling and inflammation, which are important processes in wound healing (40-43). Subsequently, tissue experiments were performed to detect the expression of several tsRNAs (tsRNA-23678, tsRNA-23727 and tsRNA-23761) and miRNAs (miR-3135b and miR-29b-1-5p). The present study revealed that tsRNAs-23678, tsRNA-23727, tsRNA-23761 and miR-3135b were upregulated while miR-29b-1-5p was downregulated in hypertrophic scar compared with normal skin tissue, which is consistent with the in vitro cell experiments. Our data showed that tsRNAs-23678, tsRNA-23727 and tsRNA-23761 belong to the 5'-tsRNA class of tsRNAs. Previous studies have found that 5'-tsRNAs can regulate the differentiation of stem cells and are involved in translational repression (44). Subsequently, the possible roles of tsRNAs-23678 and tsRNA-23761 in hypertrophic scars were revealed by target prediction, coexpression network and ceRNA analyses.

The results showed a significant upregulation of tsRNA-23761, which targets collagen type I alpha 1 chain (COL1A1). COL1A1 encodes the pro-a1 chains of type I collagen, the triple helix of which comprises two a1 chains and one a 2 chain. COL1 is a fibril-forming collagen that is found in most connective tissues and is abundant in tendons and dermis (45). Previous studies have confirmed that COL1 is the main structural element of the ECM and that its dysregulated accumulation leads to scarring (45-47). COL1 serves a critical role in the development and progression of hypertrophic scars, and its expression is increased in hypertrophic scars $(46,47)$. The present study revealed that tsRNA-23761 and COL1A1 exhibit common expression trends in hypertrophic scarring. Overall, the dysregulation of tsRNA-23761 may contribute to the formation of hypertrophic scars.

In addition, TargetScan was used to predict potential targets of tsRNA-23727, which included SMAD2. A previous study has reported that SMAD2 gene expression in hypertrophic scar fibroblasts is significantly upregulated (48), and the present study revealed that the expression of SMAD2 was positively associated with that of tsRNA-23727. The SMAD protein family is homologous to the gene products of 
Table V. Data of significant differentially expressed miRNAs of hypertrophic scar compared with normal skin $\left(\log _{2} \mathrm{FC}>1.0\right.$, $\mathrm{P}<0.05)$.

\begin{tabular}{lccc}
\hline AccID & Fold change & Log $_{2}$ FC & Regulation \\
\hline miR-29b-1-5p & 0.3214 & -1.6373 & Down \\
miR-1275 & 0.4445 & -1.7057 & Down \\
miR-148b-5p & 0.3617 & -1.4671 & Down \\
miR-151b & 0.3072 & -1.7025 & Down \\
miR-18a-5p & 0.3044 & -1.7156 & Down \\
miR-200a-3p & 0.4043 & -1.3064 & Down \\
miR-215-5p & 0.2924 & -1.7736 & Down \\
hsa-miR-222-5p & 0.4006 & -1.3196 & Down \\
miR-25-5p & 0.4175 & -1.2601 & Down \\
miR-324-5p & 0.4280 & -1.2242 & Down \\
miR-335-5p & 0.2566 & -1.9623 & Down \\
miR-491-5p & 0.3522 & -1.5054 & Down \\
miR-590-3p & 0.3644 & -1.4563 & Down \\
miR-664a-3p & 0.4117 & -1.2802 & Down \\
miR-7-1-3p & 0.3386 & -1.5621 & Down \\
miR-3135b & 2.5475 & 1.3491 & Up \\
miR-10a-5p & 39.3914 & 5.2998 & Up \\
miR-10b-5p & 57.0105 & 5.8331 & Up \\
miR-129-5p & 2.4527 & 1.2944 & Up \\
miR-133a-3p & 4.0421 & 2.0151 & Up \\
miR-137 & 2.8405 & 1.5061 & Up \\
miR-1-3p & 4.1297 & 2.0460 & Up \\
miR-140-3p & 3.5733 & 1.8372 & Up \\
miR-140-5p & 2.3318 & 1.2215 & Up \\
miR-142-3p & 10.0859 & 3.3342 & Up \\
miR-142-5p & 7.4310 & 2.8935 & Up \\
miR-143-3p & 4.9665 & 2.3122 & Up \\
miR-143-5p & 8.9891 & 3.1681 & Up \\
miR-145-3p & 4.5983 & 2.2011 & Up \\
miR-200b-3p & 3.2765 & 1.7121 & Up \\
\hline & & & \\
\hline
\end{tabular}

miR, microRNA; AccID, gene name according to miRbase.

the Drosophila mothers against decapentaplegic (Mad) and the Caenorhabditis elegans Sma genes (49). SMAD proteins are signal transducers and transcriptional modulators that mediate multiple signaling pathways (50). In a previous study, enhancement of SMAD expression promoted transforming growth factor (TGF)- $\beta 1$ secretion, and TGF- $\beta 1$ induced $\alpha$-smooth muscle actin ( $\alpha$-SMA) expression, collagen synthesis and the formation of hypertrophic scars (51). SMAD2 regulates numerous cellular processes, including proliferation, survival, apoptosis and dormancy (52-55). Altogether, the results of the present study suggest that increased expression of tsRNA-23727 promotes the development of hypertrophic scars by targeting SMAD2. This finding provides suggests that the function of tsRNAs in hypertrophic scar fibroblasts may involve the regulation of key mRNAs.

Significantly differentially expressed tsRNAs, such as tsRNA-23678, tsRNA-23761 and tsRNA-23727, in hypertrophic
Table VI. Target genes for the three differentially expressed tsRNAs.

\begin{tabular}{ll}
\hline Yingbio_ID & \multicolumn{1}{c}{ Target gene } \\
\hline tsRNA-23678 & FAS, SMAD2, ABCA1, ABCCB, ABHDS, \\
& ABL2, AIPL1, BB59 \\
tsRNA-23727 & SMAD2, TGFBR1, ABCC8, ABHD5, \\
& ABL2, BEAN1, AIPH, BAALC \\
tsRNA-23761 & COL1A1, A1CF, ABI3, BEST1, \\
& ABCA1, ACAP3, ACE, B3GNT7
\end{tabular}

tsRNA, tRNA-derived small RNAs.

Table VII. Comparison of RT-qPCR data with RNA-seq data for tsRNAs.

\begin{tabular}{lccc}
\hline & \multicolumn{3}{c}{ HA/HB ratio } \\
\cline { 2 - 4 } Method & tsRNA-23678 & tsRNA-23727 & tsRNA-23761 \\
\hline RT-qPCR & 1.9452 & 1.6684 & 1.6997 \\
RNA-seq & 5.2361 & 8.8052 & 5.9145 \\
\hline
\end{tabular}

RT-qPCR, reverse transcription-quantitative PCR; RNA-seq, RNA sequencing; tsRNA, tRNA-derived small RNA; HA, hypertrophic scar; HB, normal skin.

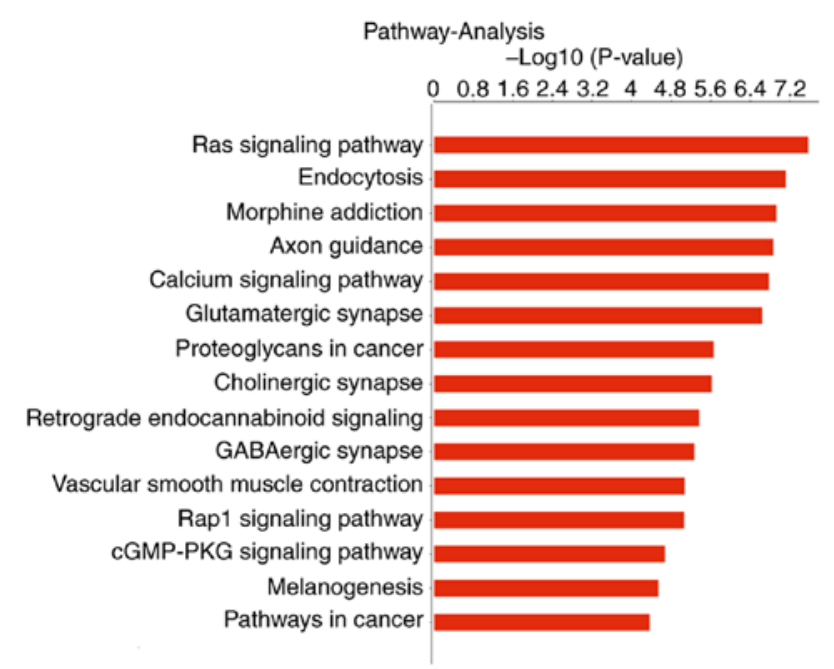

Figure 3. KEGG pathway analysis of differentially expressed tsRNAs. The top 15 enriched pathways of tsRNAs in the KEGG analysis are presented. $-\log _{10}$ represents the $-\log _{10}$ of the P-value, and a smaller P-value is indicated by a larger $-\log _{10}$ P-value. KEGG, Kyoto Encyclopedia of Genes and Genomes; tsRNAs, tRNA-derived small RNAs.

scars were selected for construction of an miRNA-tsRNA coexpression network in the present study according to the degree of correlation. Functional prediction of tsRNAs can be based on annotation of the function of coexpressed miRNAs. In the present study, analyses using TargetScan and miRanda databases were performed to identify miRNAs with complementarity to the selected tsRNAs. tsRNA-23678 was 
A Amplification plot

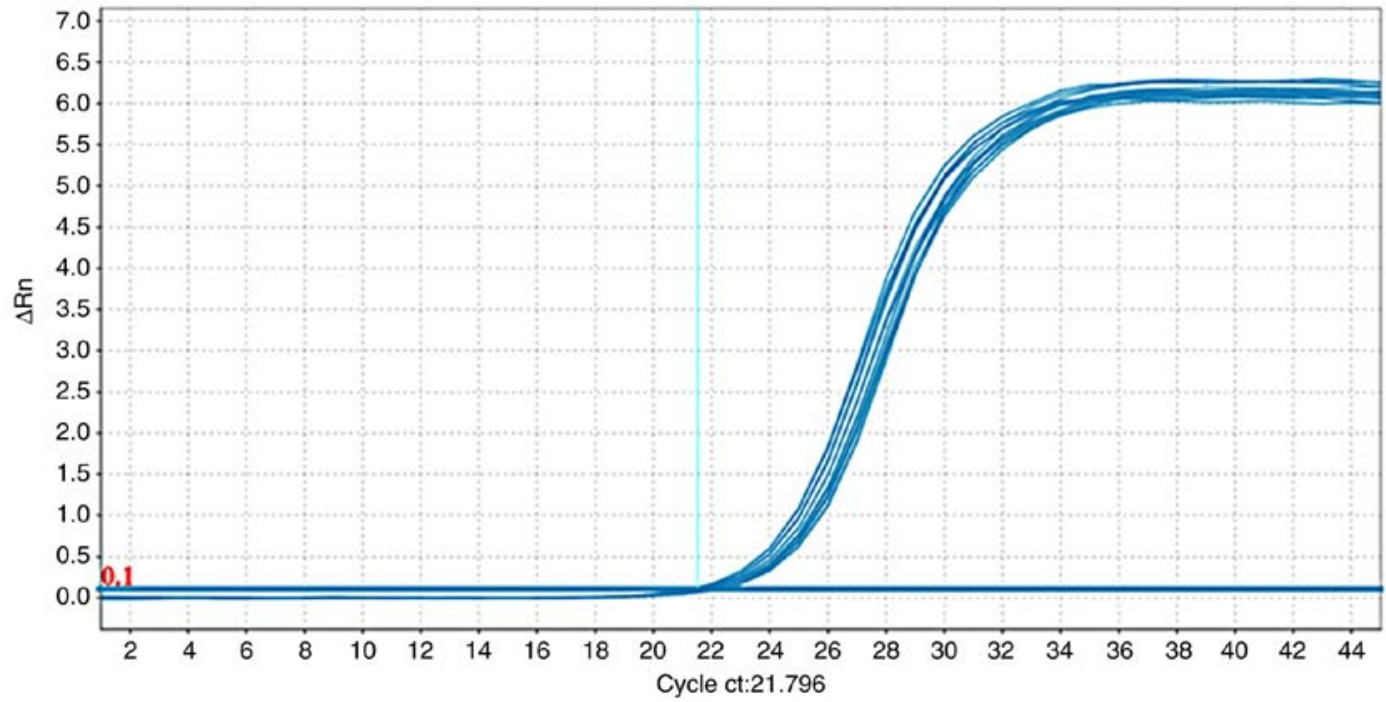

B

Amplification plot

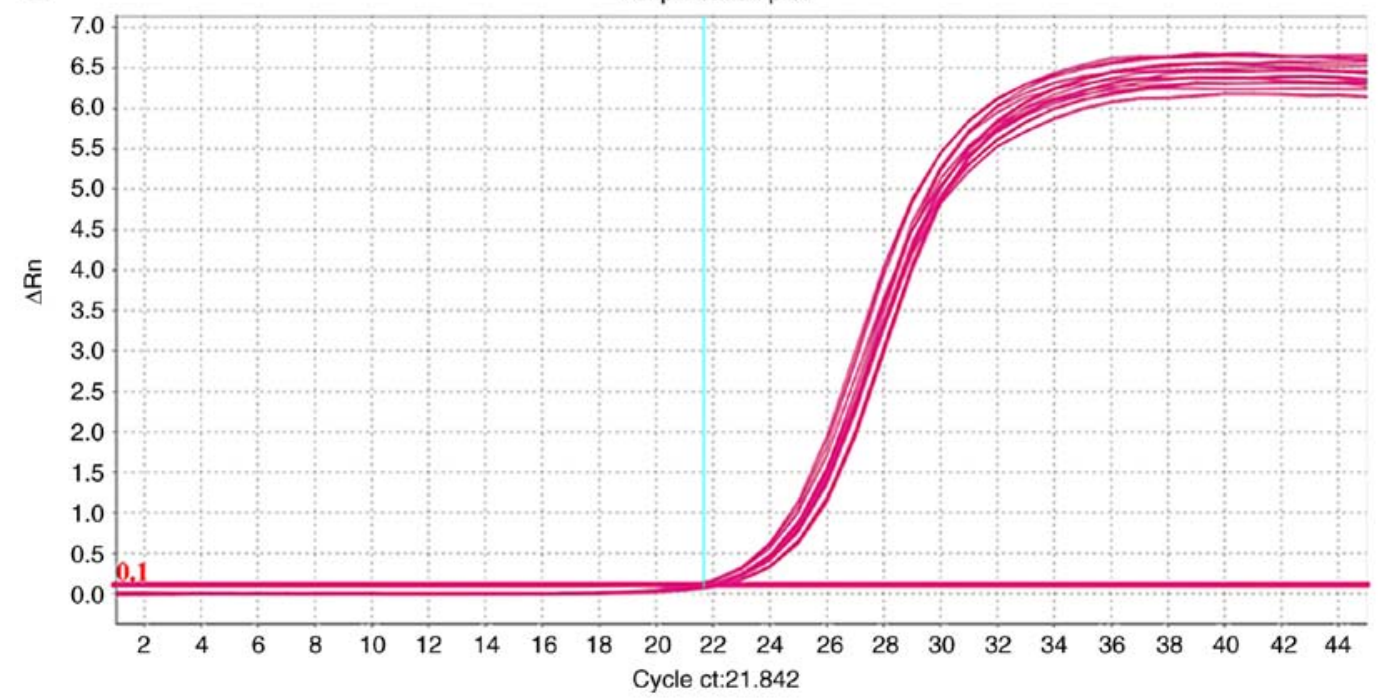

C

Amplification plot

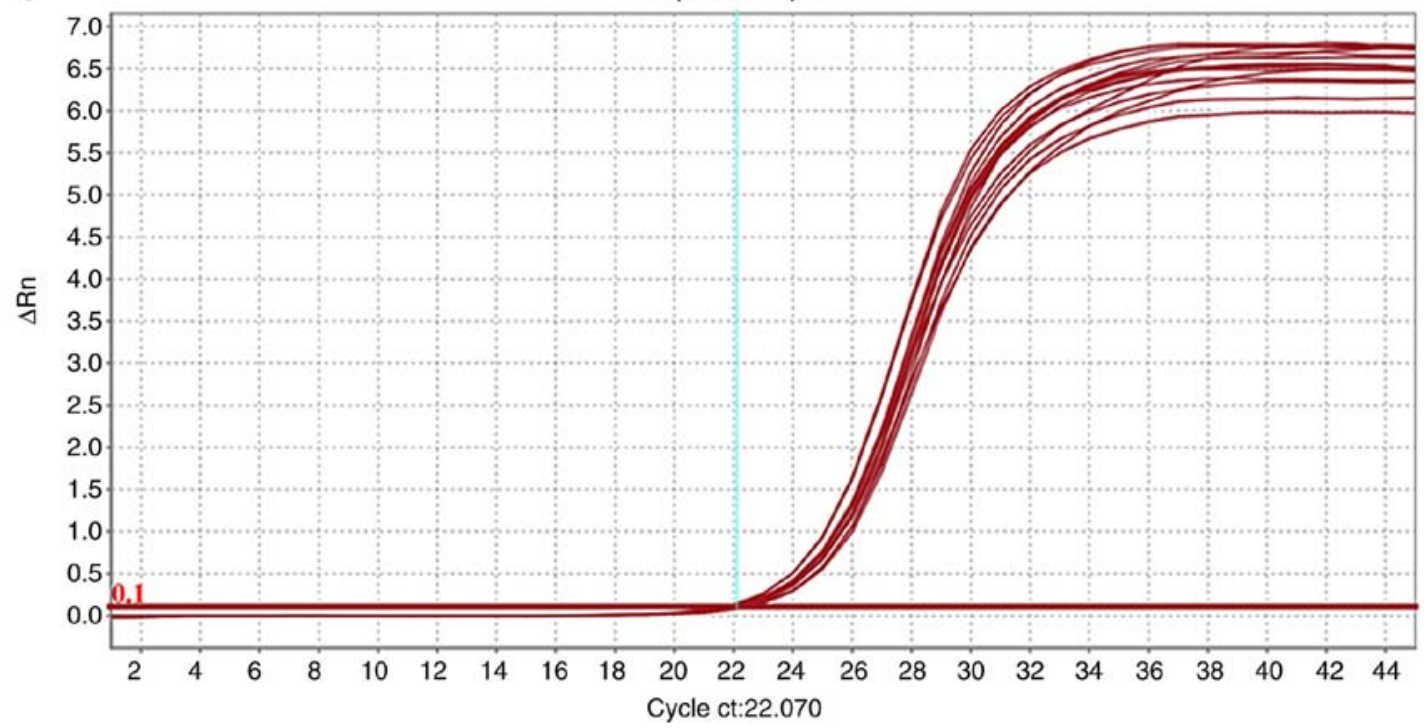

Figure 4. Amplification plots and dissociation curves for selected tsRNAs. Amplification plots for (A) tsRNA-23678, (B) tsRNA-23727, (C) tsRNA-23761. In the amplification curves, the $\mathrm{x}$-axis represents the cycle number, and the $\mathrm{y}$-axis represents the real-time fluorescence signal intensity of the corresponding cycle number. In the dissociation curves, the x-axis represents the temperature of the RT-qPCR products, and the y-axis represents the real-time fluorescence-signal-intensity change rate with increasing temperature. Differently colored curves correspond to different RT-qPCRs. tsRNAs, tRNA-derived small RNA; RT-qPCR, reverse transcription-quantitative PCR. 

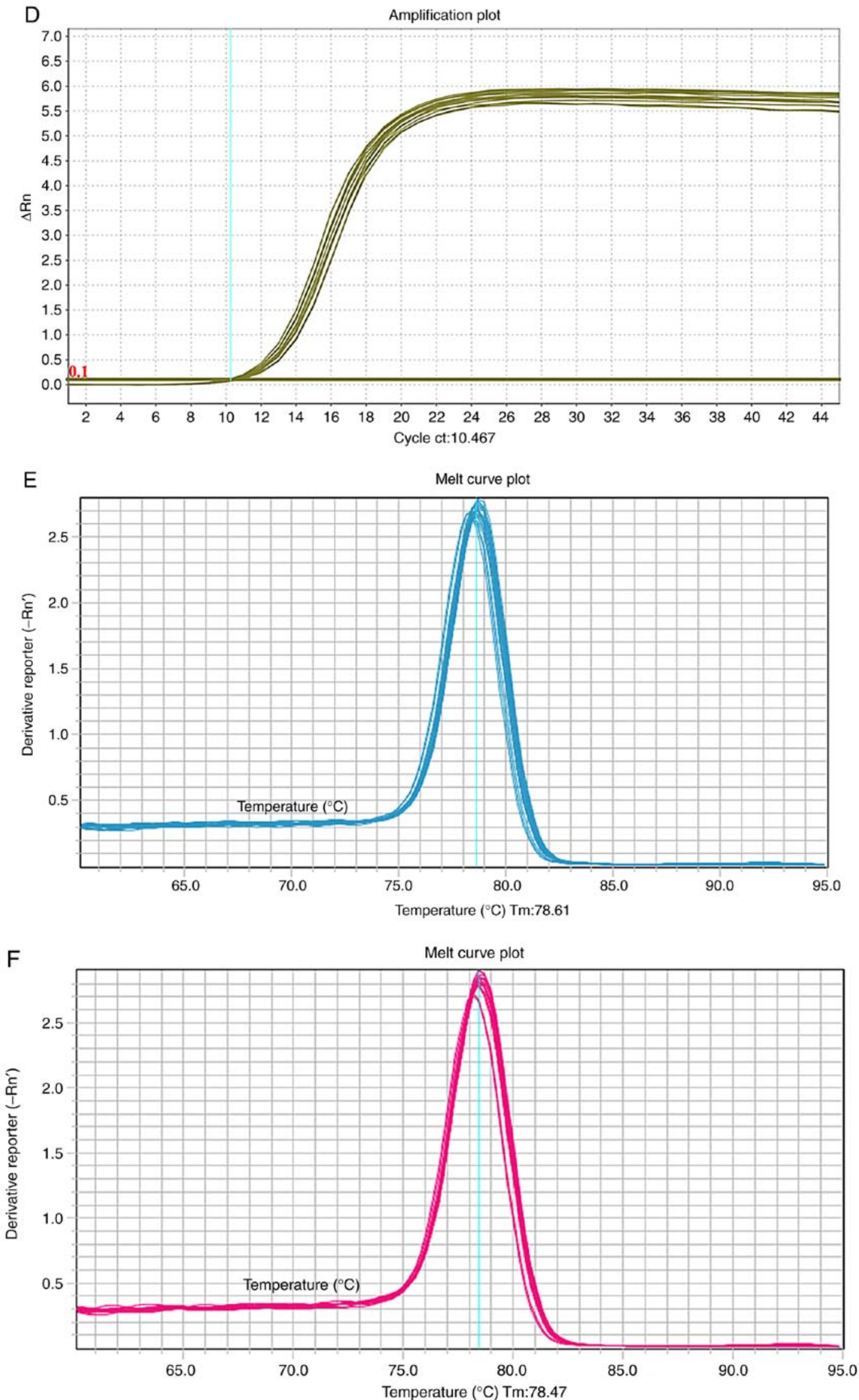

Figure 4. Continued. Amplification plots and dissociation curves for selected tsRNAs. Amplification plots for (D) U6. Dissociation curves for (E) tsRNA-23678, (F) tsRNA-23727. In the amplification curves, the $\mathrm{x}$-axis represents the cycle number, and the $\mathrm{y}$-axis represents the real-time fluorescence signal intensity of the corresponding cycle number. In the dissociation curves, the $\mathrm{x}$-axis represents the temperature of the RT-qPCR products, and the y-axis represents the real-time fluorescence-signal-intensity change rate with increasing temperature. Differently colored curves correspond to different RT-qPCRs. tsRNAs, tRNA-derived small RNA; RT-qPCR, reverse transcription-quantitative PCR. 

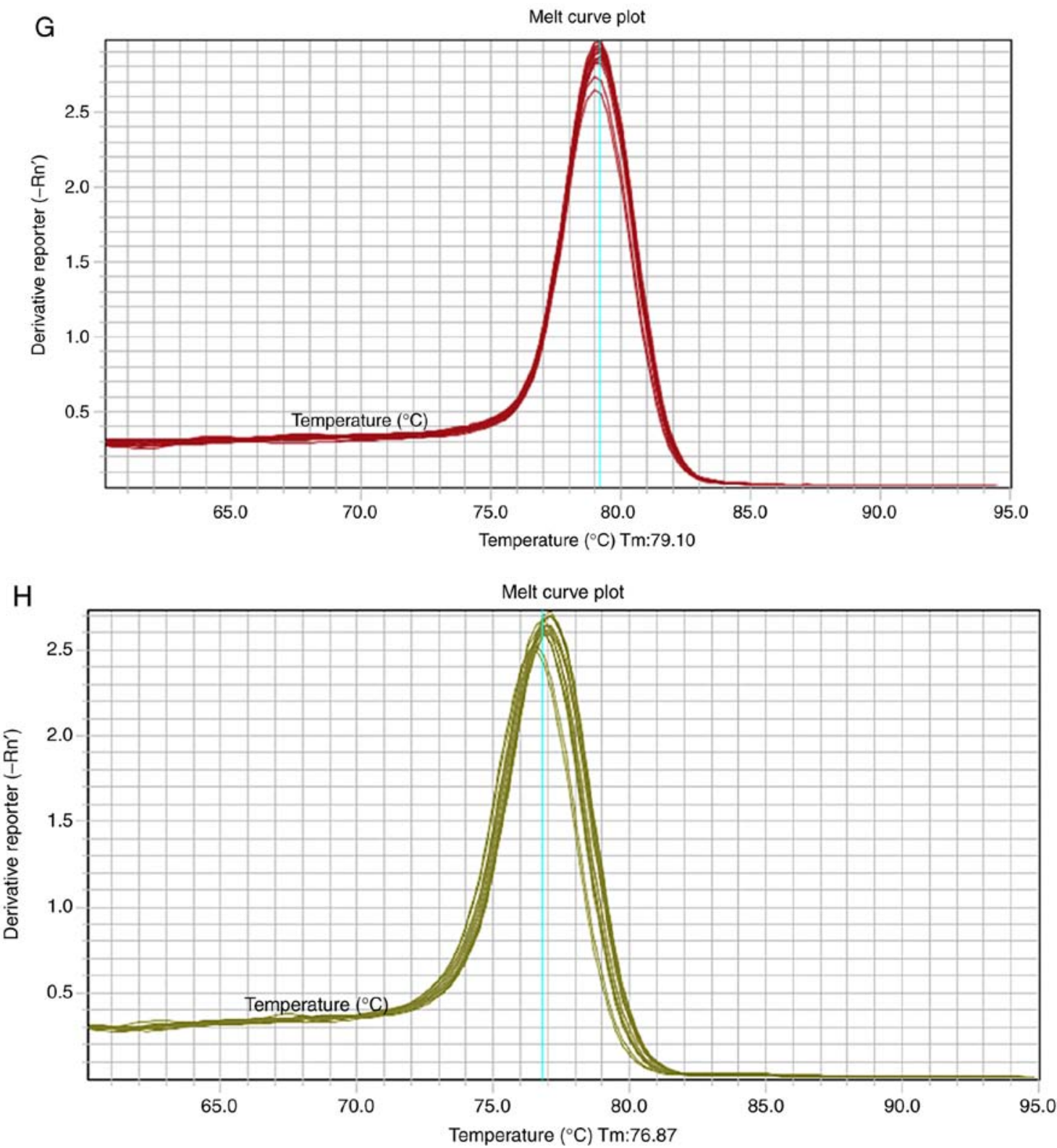

Figure 4. Continued. Amplification plots and dissociation curves for selected tsRNAs. Amplification plots for (G) tsRNA-23761 and (H) U6. In the amplification curves, the $\mathrm{x}$-axis represents the cycle number, and the $\mathrm{y}$-axis represents the real-time fluorescence signal intensity of the corresponding cycle number. In the dissociation curves, the $\mathrm{x}$-axis represents the temperature of the RT-qPCR products, and the y-axis represents the real-time fluorescence-signal-intensity change rate with increasing temperature. Differently colored curves correspond to different RT-qPCRs. tsRNAs, tRNA-derived small RNA; RT-qPCR, reverse transcription-quantitative PCR.

complementary to multiple miRNAs, including miR-1268b, miR-1303, miR-1275, miR-17-5p, miR-15b-5p, miR-29b-1-5p, miR-222-3p, miR-423-5p, miR-491-5p, miR-423-5p, miR-3135b, miR-143-5p and miR-133a-3p. These miRNAs are implicated in a number of biological processes, such as cell proliferation, the cell cycle, apoptosis and angiogenesis. Some important related miRNAs are known to be associated with hypertrophic scars, such as miR-29b. Indeed, a previous study reported that miR-29b has anti-fibrosis activity, and demonstrated that the overexpression of miR-29b significantly reduced the expression levels of COL1A1 and $\alpha$-SMA, inhibited cell proliferation and induced apoptosis in hypertrophic scar fibroblasts compared with normal fibroblasts (56). The miR-29b mimic remlarsen may be an effective treatment for preventing fibrotic scars (hypertrophic scars or keloids) from forming, or for preventing skin fibrosis such as scleroderma (57). miR-29 can directly inhibit YY1 and TGF- $\beta 3$, improve skeletal muscle atrophy and reduce renal fibrosis by the lowering levels of YY1 and TGF- $\beta$ pathway proteins (58). Consistent with this, Bi et al (45) reported that the miR-29 family plays a role in hypertrophic scars by regulating the translation of ECM mRNA. In addition, the data from the present study demonstrated that tsRNA-23678 was upregulated in hypertrophic scar compared with normal skin, while the expression of miR-29b-1-5p, a member of the miR-29b family, was markedly lower, which indicated opposing effects. The coexpression network of miRNA-tsRNA indicated that tsRNA-23678 was complementary to miR-29b-1-5p. Functional prediction of tsRNA-23678 can be based on annotation of the function of the coexpressed miR-29b-1-5p. These findings suggest that 


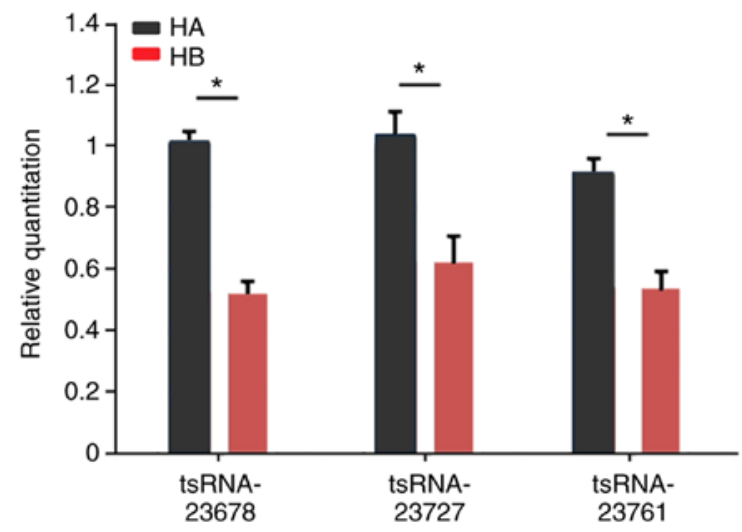

Figure 5. Verification of the expression of significantly dysregulated tsRNAs by reverse transcription-quantitative PCR. Relative quantitative levels of three tsRNAs were detected in HA and HB groups. Data are presented as the mean $\pm \mathrm{SD}(\mathrm{n}=3) ;{ }^{*} \mathrm{P}<0.05$ as indicated. tsRNAs, tRNA-derived small RNA; HA, hypertrophic scar tissues; HB, normal tissues.

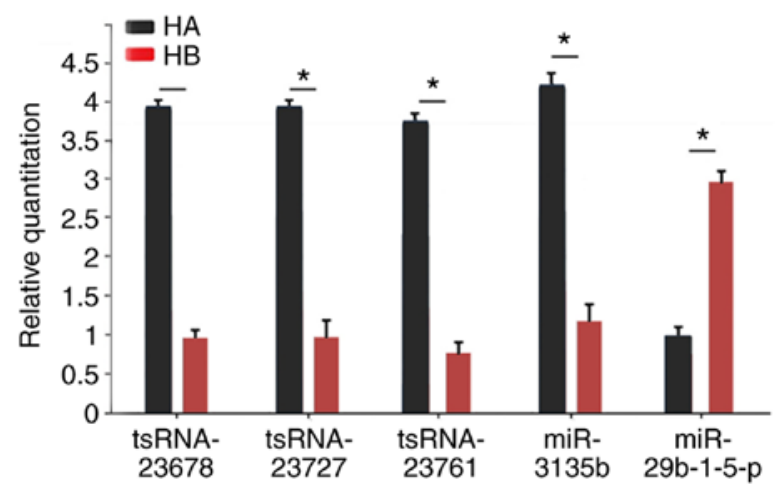

Figure 6. Reverse transcription-quantitative PCR analysis for several tsRNAs and miRNAs in hypertrophic scar tissue and normal skin tissue, to verify the in vitro experimental results. Data are presented as the mean $\pm \mathrm{SD}(\mathrm{n}=3) ;{ }^{*} \mathrm{P}<0.05$ as indicated. tsRNAs, tRNA-derived small RNA; miRNA, microRNA; HA, hypertrophic scar tissues; HB, normal tissues.

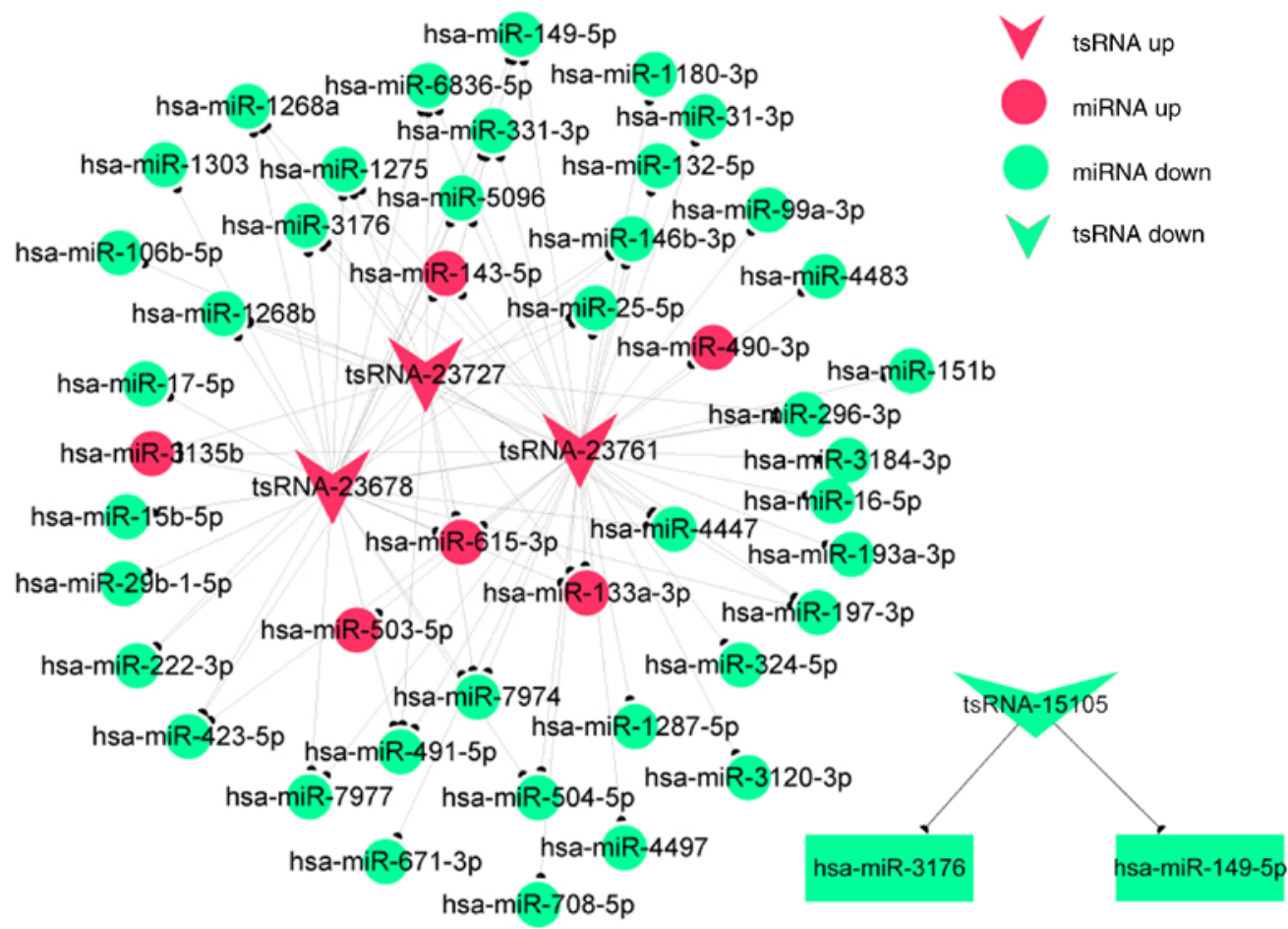

Figure 7. Coexpression networks of three significantly dysregulated tsRNAs with their associated miRNAs in hypertrophic scars. Red indicates upregulated genes, and green indicates downregulated genes. tsRNAs, tRNA-derived small RNA; miRNA, microRNA.

tsRNA-23678 may be an important regulator of hypertrophic scars. A tsRNA-miRNA-mRNA expression network based on RNA-seq data was constructed to further investigate the mechanisms underlying tsRNAs. This network indicated the predicted tsRNA-miRNA-mRNA associations in hypertrophic scars and included three tsRNAs, six miRNAs and 40 mRNAs. Recent studies have shown that tsRNAs can be used directly as miRNAs to identify target mRNA, both acting on mRNA and inhibiting target genes through translation $(59,60)$. The present study indicated that tsRNA acts as a ceRNA. It is known that miRNA can lead to gene silencing by binding to mRNA, whereas ceRNA regulates gene expression by competing with miRNA (61). The ceRNA hypothesis indicates that RNA molecules harboring MREs can communicate with each other by competing with miRNA. Understanding this novel RNA crosstalk may provide insight into the function of tsRNAs (62). The ceRNA network in the present study showed that tsRNA-23761 may act as a competing endogenous RNA that binds miR-3135b to regulate the expression of miR targets, including ACE. The present high-throughput sequencing analysis demonstrated tsRNA-23761 to be upregulated in hypertrophic scars; associated miR-3135b and ACE were also upregulated, indicating a similar trend. The ACE gene, located in the $17 \mathrm{q} 23.3$ position of chromosome 17, encodes angiotensin I-converting enzyme. The full-length ACE gene comprises 44,769 bp, and the full-length ACE mRNA 


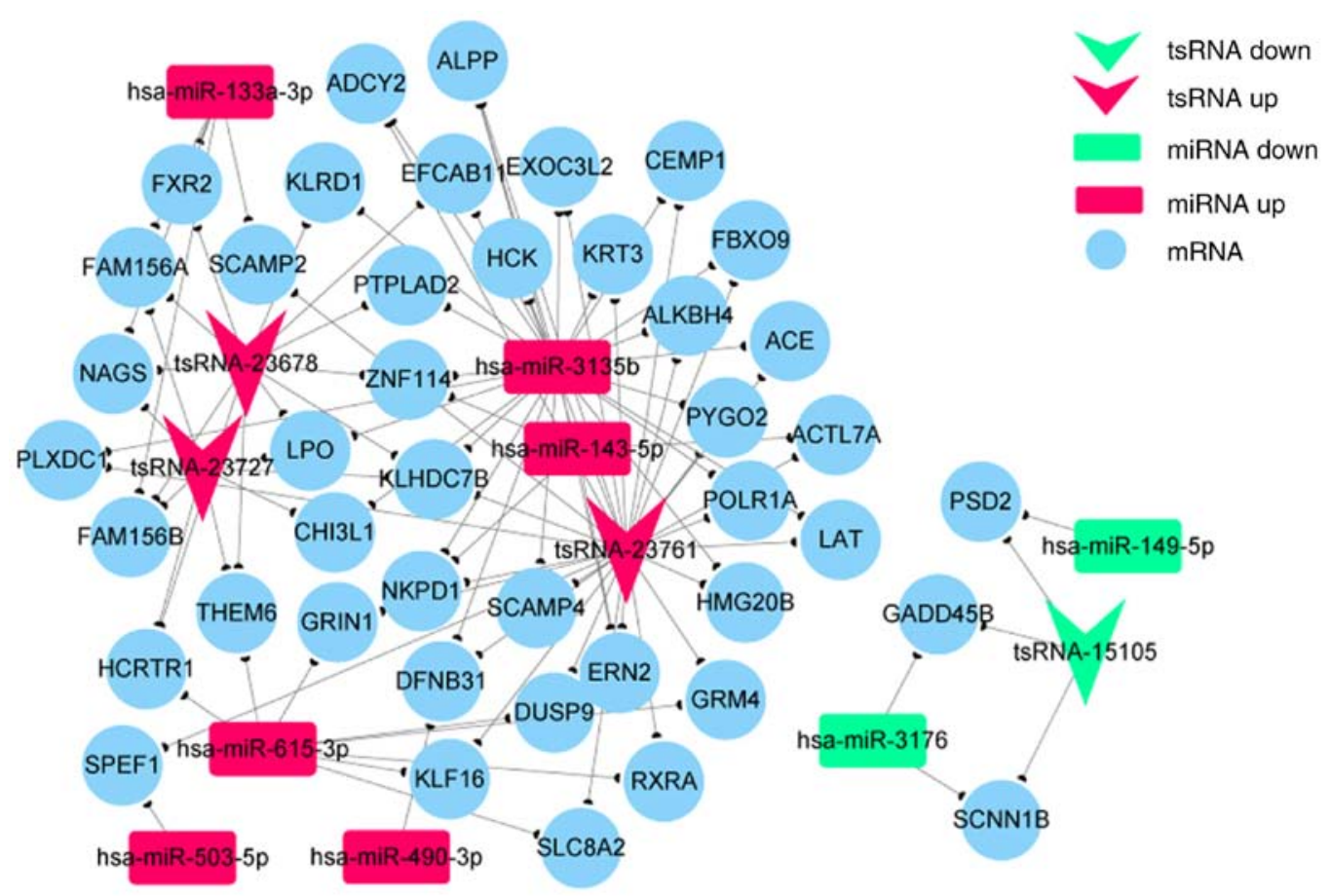

Figure 8. tsRNA-miRNA-mRNA network in hypertrophic scars, based on tsRNA-miRNA and mRNA-miRNA interactions. In this network, tsRNA aRNA; miRNA, microRNA.

comprises $4,195 \mathrm{nt}$; the ACE gene harbors a total of 48 exons and encodes 1,306 amino acid residues. Angiotensin II activation by ACE is a significant mediator in wound healing and collagen production. Previous studies have shown that the expression of ACE in scars is significantly higher than that in normal skin, inducing the conversion of angiotensin I to angiotensin II, leading to excessive deposition of ECM and fibrosis, and regulating the activity and proliferation of fibroblasts in proliferative scars (63). ACE is an important regulatory factor for the renin angiotensin aldosterone system and the bradykinin system, which affect many physiological functions of the human body (64). tsRNA-23761 competes with the miRNA pool to regulate ACE expression. It is speculated that tsRNA-23761 may also affect the activity and proliferation of fibroblasts in hypertrophic scars via the regulation of certain mechanisms of ACE (63-65).

In summary, with the development of bioinformatics technology, it has been discovered that tsRNAs serve important roles in the occurrence and development of disease. However, the functions of the majority of tsRNAs have not been annotated to date. In the present study, tsRNAs that were differentially expressed in fibroblasts and tissue derived from hypertrophic scar tissue compared with normal skin tissue were identified. The data indicate that tsRNA-23678, tsRNA-23727 and tsRNA-23761 were significantly upregulated in fibroblasts and tissue derived from hypertrophic scar compared with normal skin tissue. tsRNA-23678 may serve critical functions through interaction with miR-29b-1-5p to modulate hypertrophic scars. In addition, tsRNA-23761 may interact with mRNA, such as ACE, to affect the activity and proliferation of fibroblasts in hypertrophic scars. As hypertrophic scar development is a dynamic process that is affected by many factors, the results of the present study are preliminary and limited. Further in vivo experiments are required to confirmed the results.
The significantly differentially expressed tsRNAs identified in the present study may contribute to our understanding of the potential mechanisms of hypertrophic scars, as well as therapeutic strategies.

\section{Acknowledgements}

The authors would like to thank Professor Jianhua Fu from the First Affiliated Hospital of Nanchang University for providing samples for analysis in this study.

\section{Funding}

The present study was supported by grants from the National Natural Science Foundation of China (grant no. 81860340) and the special fund for the Graduate Innovation Project of Nanchang University (grant no. CX2019166).

\section{Availability of data and materials}

The datasets used during the current study are available from the corresponding author on reasonable request.

\section{Authors' contributions}

YZ and DL designed the study. YZ, QD, LT and DL performed the experiments. YZ was also involved in data analysis and completed the manuscript editing with the help of DL. All authors read and approved the final version of the manuscript.

\section{Ethics approval and consent to participate}

This study was approved by the Ethics Committee of the First Affiliated Hospital of Nanchang University, and written 
informed consent was obtained from all participants or their parents/legal guardians.

\section{Patient consent for publication}

Not applicable.

\section{Competing interests}

The authors declare that they have no competing interests.

\section{References}

1. Bayat A, McGrouther DA and Ferguson MW: Skin scarring. BMJ 326: 88-92, 2003.

2. Sidgwick GP, Iqbal SA and Bayat A: Altered expression of hyaluronan synthase and hyaluronidase mRNA may affect hyaluronic acid distribution in keloid disease compared with normal skin. Exp Dermatol 22: 377-379, 2013

3. Syed F, Ahmadi E, Iqbal SA, Singh S, McGrouther DA and Bayat A: Fibroblasts from the growing margin of keloid scars produce higher levels of collagen I and III compared with intralesional and extralesional sites: Clinical implications for lesional site-directed therapy. Br J Dermatol 164: 83-96, 2011.

4. Wolfram D, Tzankov A, Pulzl P and Piza-Katzer H: Hypertrophic scars and keloids-a review of their pathophysiology, risk factors, and therapeutic management. Dermatol Surg 35: 171-181, 2009.

5. Qi J, Liu Y, Hu K, Zhang Y, Wu Y and Zhang X: MicroRNA-26a inhibits hyperplastic scar formation by targeting Smad2. Exp Ther Med 15: 4332-4338, 2018.

6. Zhu Z, Ding J, Shankowsky HA and Tredget EE: The molecular mechanism of hypertrophic scar. J Cell Commun Signal 7: 239-252, 2013

7. van der Veer WM, Bloemen MC, Ulrich MM, Molema G, van Zuijlen PP, Middelkoop E and Miessen FB: Potential cellular and molecular causes of hypertrophic scar formation. Burns 35 : 15-29, 2009.

8. Rumsey N, Clarke A and White P: Exploring the psychosocial concerns of outpatients with disfiguring conditions. J Wound Care 12: 247-252, 2003

9. Leblebici B, Adam M, Bagis S, Tarim AM, Noyan T, Akman MN and Haberal MA: Quality of life after burn injury: The impact of joint contracture. J Burn Care Res 27: 864-868, 2006.

10. Zuccaro J, Ziolkowski N and Fish J: A systematic review of the effectiveness of laser therapy for hypertrophic burn scars. Clin Plast Surg 44: 767-779, 2017.

11. Li S, Xu Z and Sheng J. tRNA-Derived small RNA: A novel regulatory small non-coding RNA. Genes (Basel) 9: E246, 2018

12. Anderson $P$ and Ivanov $P$ : tRNA fragments in human health and diease. FEBS Lett 588: 4297-4304, 2014.

13. Pliatsika V, Loher P, Telonis AG and Rigoutsos I: MINTbase: A framework for the interactive exploration of mitochondrial and nucleartRNA fragments. Bioinformatics 32: 2481-2489, 2016.

14. Zheng LL, Xu WL, Liu S, Sun WJ, Li JH, Wu J, Yang JH and Qu LH: tRFCancer: A web server to detect tRNA-derived smal RNA fragments (tRFs) and their expression in multiple cancers. Nucleic Acids Res 44: W185-W193, 2016.

15. Lee YS, Shibata Y, Malhotra A and Dutta A: A novel class of small RNAs: tRNA-derived RNA fragments (tRFs). Genes Dev 23: 2639-2649, 2009

16. Wang Q, Lee I, Ren J, Ajay SS, Lee YS and Bao X: Identification and functional characterization of tRNA-derived RNA fragments (tRFs) in respiratory syncytial virus infection. Mol Ther 21: 368-379, 2013.

17. Tomita K, Ogawa T, Uozumi T, Watanabe K and Masaki H: A cytotoxic ribonuclease which specifically cleaves four isoaccepting arginine tRNAs at their anticodon loops. Proc Natl Acad Sci USA 97: 8278-8283, 2000.

18. Deng J, Ptashkin RN, Wang Q, Liu G, Zhang G, Lee I, Lee YS and Bao X: Human metapneumovirus infection induces significant changes in small noncoding RNA expression in airway epithelial cells. Mol Ther Nucleic Acids 3: e163, 2014.

19. Gonzale N and Goleberg DJ: Update on the treatment of scars. J Drugs Dermatol 18: 550, 2019.

20. Ault P, Plaza A and Paratz J: Scar massage for hypertrophic burns scarring-A systematic review. Burns 44: 24-38, 2018.
21. Tu L, Huang Q, Fu S and Liu D: Aberrantly expressed long noncoding RNAs in hypertrophic scar fibroblast in vitro: A microarray study. Int J Mol Med 41: 1917-1930, 2018.

22. Liu Y, Zhong L, Liu D, Ye H, Mao Y and Hu Y: Differential miRNA expression profiles in human keratinocytes in response to protein kinase $\mathrm{C}$ inhibitor. Mol Med Rep 16: 6608-6619, 2017.

23. Chen MH, Chen YJ, Ye YQ, Su Y and Li CY: The factors affecting analysis of gene expression level by semi qRT-PCR technique. Guzhou Agricultural Sciences 29: 28-30,33, 2009.

24. Lowe TM and Eddy SR: tRNAscan-SE: A program for improved detection of transfer RNA genes in genomic sequence. Nucleic Acids Res 25: 955-964, 1997.

25. Kumar P, Mudunuri SB, Anaya J and Dutta A: tRFdb: A database for transfer RNA fragments. Nucleic Acids Res 43: D114-D115, 2015.

26. Pliatsika V, Loher $\mathrm{P}$, Magee R, Telonis AG, Londin E, Shigematsu M, Kirino Y and Rigoutsos I: MINTbase v2.0: A comprehensive database for tRNA-derived fragments that includes nuclear and mitochondrial fragments from all The Cancer Genome Atlas projects. Nucleic Acids Res 46: D152-D159, 2018.

27. Conesa A, Madrigal P, Tarazona S, Gomez-Cabrero D, Cervera A, McPherson A, Szcześniak MW, Gaffney DJ, Elo LL, Zhang X and Mortazavi A: A survey of best practices for RNA-seq data analysis. Genome Biol 17: 13, 2016.

28. Wang L, Feng Z, Wang X and Zhang X: DEGseq: An R package for identifying differentially expressed genes for RAN-seq data. Bioinformatics 26: 136-138, 2009.

29. Adnan M, Morton G and Hadi S: Analysis of rpoS and bolA gene expression under various 0stress-induced environments in planktoic and biofilm phase using $2(-\Delta \Delta \mathrm{CT})$ method. Mol Cell Biochem 357: 275-282, 2011.

30. Kim HK, Fuchs G, Wang S, Wei W, Zhang Y, Park H, Roy-chaudhuri B, Li P, Xu J, Chu K, et al: A transfer-RNA-derived small RNA regulates ribosome biogenesis. Nature 552: 57-62, 2017.

31. Kumar P, Anaya J, Mudunuri SB and Dutta A: Meta-analysis of tRNA derived RNA fragments reveals that they are analysis of tRNA derived RNA fragments reveals that they are evolutionarily conserved and associate with AGO proteins to recognize specific RNA targets. BMC Biol 12: 78, 2014.

32. Keam SP and Hutvagner G: tRNA-Derived fragments (tRFs): Emerging new roles for an ancient RNA in the regulation of gene expression. Life (Basel) 5: 1638-1651, 2015.

33. Balatti V, Pekarsky Y and Croce CM: Role of the tRNA-derived Small RNAs in cancer: New potential biomarkers and target for therapy. Adv Cancer Res 135: 173-187, 2017.

34. Maute RL, Schneider C, Sumazin P, Holmes A, Califano A, Basso $\mathrm{K}$ and Dalla-Favera R: tRNA-derived microRNA modulates proliferation and the DNA damage response and is down-regulated in B cell lymphoma. Proc Natl Acad Sci USA 110: 1404-1409, 2013.

35. Sobala A and Hutvagner G: Small RNAs derived from the 5' end of tRNA can inhibit protein translation in human cells. RNA Biol 10: 553-563, 2013.

36. Gebetsberger J,Zywicki M,Kunzi A and PolacekN:tRNA-derived fragments target the ribosome and function as regulatory non-coding RNA in Haloferax volcanii. Archaea 2012: 260909, 2012.

37. Goodarzi H, Liu X, Nguyen HC, Zhang S, Fish L and Tavazoie SF: Endogenous tRNA-derived fragments suppress breast cancer progression via YBX1 displacement. Cell 161: 790-802, 2015.

38. Kumar P, Kuscu C and Dutta A: Biogenesis and function of transfer RNA-related fragments (tRFs). Trends Biochem Sci 41: 679-689, 2016

39. Atiyeh BS: Nonsurgical management of hypertrophic scars: Evidence-based therapies, standardpractices, and emerging methods. Aesthetic Plast Surg 31: 468-492, 2007.

40. Nehme A, Cerutti C, Dhaouadi N, Gustin MP, Courand PY, Zibara K and Bricca G: Atlas of tissue renin-angiotensin-aldosterone system in human: A transcriptomic meta-analysis. Sci Rep 5: 10035, 2015.

41. Slack C: Ras signaling in aging and metabolic regulation. Nutr Healthy Aging 4: 195-205, 2017.

42. Koul HK, Pal M and Koul S: Role of p38 MAP kinase signal transduction in solid tumors. Genes Cancer 4: 342-359, 2013.

43. Coelho MA, de Carne Trecesson S, Rana S, Zecchin D, Moore C, Molina-Arcas M, East P, Spencer-Dene B, Nye E, Barnouin K, et al: Oncogenic RAS signaling promotes tumor immunoresistance by stabilizing PD-L1 mRNA. Immunity 47: 1083-1099 e6, 2017. 
44. Krishna S, Yim DG, Lakshmanan V, Tirumalai V, Koh JL, Park JE, Cheong JK, Low JL, Lim MJ, Sze SK, et al: Dynamic expression of tRNA-derived small RNAs define cellular states. EMBO Rep 20: e47789, 2019.

45. Bi S, Cao C, Chai LL, Li SR and Yang DY: Regulatory mechanism of miR-29 over TGF- $\beta 1$ and COL1 in scar cells. Eur Rev Med Pharmacol Sci 21: 2512-2517, 2017.

46. Xiao K, Luo X, Wang X and Gao Z: MicroRNA185 regulates transforming growth factor- $\beta 1$ and collagen 1 in hypertrophic scar fibroblasts. Mol Med Rep 15: 1489-1496, 2017.

47. Zeng G, Zhong F, Li J, Luo S and Zhang P: Resveratrol-mediated reduction of collagen by inhibiting proliferation and producing apoptosis in human hypertrophic scar fibroblasts. Biosci Biotechnol Biochem 77: 2389-2396, 2013.

48. Qi J,Liu Y,HuK,Zhang Y,Wu Y and Zhang X: MicroRNA-205-5p regulates extracellular matrix production in hyperplastic scars by targeting Smad2. Exp Ther Med 17: 2284-2290, 2019.

49. Li Q: Inhibitory SMADs: Potential regulators of ovarian function. Biol Reprod 92: 50, 2015.

50. Zhang Y, Alexander PB and Wang XF: TGF- $\beta$ family signaling in the control of cell proliferation and survival. Cold Spring Harb Perspect Biol 9: a022145, 2017.

51. Zhang Z, Kuang F, Liu CL, Chen B, Tang WB and Li XJ: Effects of silencing Smad ubiquitination regulatory factor 2 on the function of human hypertrophic scar-derived fibroblasts. Zhonghua Shao Shang Za Zhi 33: 145-151, 2017 (In Chinese).

52. Asnaghi L, White DT, Key N, Choi J, Mahale A, Alkatan H Edward DP, Elkhamary SM, Al-Mesfer S, Maktabi A, et al: ACVR1C/SMAD2 signaling promotes invasion and growth in retinoblastoma. Oncogene 38: 2056-2075, 2019.

53. Huang $\mathrm{C}, \mathrm{Wu}$ XF and Wang XL: Trichostatin Ainhibits phenotypic transition and induces apoptosis of the TAF-treated normal colonic epithelial cells through regulation of TGF- $\beta$ pathway. Int J Biochem Cell Biol 114: 105565, 2019.

54. Yamazaki S and Nakauchi H: Bone marrow Schwann cells induce hematopoietic stem cell hibernation. Int J Hematol 99: 695-698, 2014.

55. LIU N, Li Y, SUN L, JIANG J and ZHANG J: Expressions of Smad 2 and Smad4 proteins in breast carcinoma tissue and significances. J Jilin University (Medicine Edition) 42: 763-767, 2016.

56. Li J, Cen B, Chen S and He Y: MicroRNA-29b inhibits TGF- $\beta 1$-induced fibrosis via regulation of the TGF- $\beta 1 / \mathrm{Smad}$ pathway in primary human endometrial stromal cells. Mol Med Rep 13: 4229-4237, 2016.
57. Gallant-Behm CL, Piper J, Lynch JM, Seto AG, Hong SJ, Mustoe TA, Maari C, Pestano LA, Dalby CM,Jackson AL, et al: A MicroRNA-29 mimic (Remlarsen) represses extracellular matrix expression and fibroplasia in the skin. J Invest Dermatol 139: 1073-1081, 2019.

58. Wang H, Wang B, Zhang A, Hassounah F, Seow Y, Wood M, Ma F, Klein JD, Price SR and Wang XH: Exosome-mediated miR-29 transfer reduces muscle atrophy and kidney fibrosis in mice. Mol Ther 27: 571-583, 2019.

59. Luo S, He F, Luo J, Dou S, Wang Y, Guo A and Lu J: Drosophila tsRNAs preferentially suppress general translation machinery via antisense pairing and participate in cellular starvation response. Nucleic Acids Res 46: 5250-5268, 2018.

60. Schopman NC, Heynen S, Haasnoot J and Berkhout B: A miRNA-tRNA mix-up: TRNA origin of proposed miRNA. RNA Biol 7: 573-576, 2010.

61. Li M, Liu DW and Lei W: Advances in the research of effects of competing endogenous RNAs and their regulatory networks in pathological scars of skin. Zhonghua Shao Shang Za Zhi 35: 701-704, 2019 (In Chinese).

62. Tay Y, Rinn J and Pandolfi PP: The multilayered complexity of ceRNA crosstalk and competition. Nature 505: 344-352, 2014.

63. Zhang YJ, Yang XS, Wu PS, Li X, Zhang XF, Chen XQ and YU ZX: Effects of angiotensin II and losartan on the growth and proliferation of hepatic stellate cells. Di Yi Jun Yi Da Xue Xue Bao 23: 219-221, 2003.

64. Wang R, Chen J, Zhang Z and Cen Y: Role of chymase in the local renin-angiotensin system in keloids: Inhibition of chymase may be an effective therapeutic approach to treat keloids. Drug Des Devel Ther 9: 4979-4988, 2015.

65. Demir CY, Ersoz ME, Erten R, Kocak OF, Sultanoglu Y and Basbugan Y: Comparison of enalapril, candesartan and intralesional triamcinolone in reducing hypertrophic scar development: An experimental study. Aesthetic Plast Surg 42: 352-361, 2018.

This work is licensed under a Creative Commons Attribution-NonCommercial-NoDerivatives 4.0 International (CC BY-NC-ND 4.0) License. 